\title{
PCMDI
}

UCRL-ID-120121

\section{Hints on the Use of the PCMDI Visualization and Computation System (VCS)}

by

Dean N. Williams, Robert L. Mobley, . Robert S. Drach, and Thomas J. Phillips

Program for Climate Model Diagnosis and Intercomparison Lawrence Livermore National Laboratory, Livermore, CA, USA

April 1995 


\section{DISCLAIMER}

This report was prepared as an account of work sponsored by an agency of the United States Government. Neither the United States Government nor any agency thereof, nor any of their employees, make any warranty, express or implied, or assumes any legal liability or responsibility for the accuracy, completeness, or usefulness of any information, apparatus, product, or process disclosed, or represents that its use would not infringe privately owned rights. Reference herein to any specific commercial product, process, or service by trade name, trademark, manufacturer, or otherwise does not necessarily constitute or imply its endorsement, recommendation, or favoring by the United States Government or any agency thereof. The views and opinions of authors expressed herein do not necessarily state or reflect those of the United States Government or any agency thereof. 


\section{DISCLAIMER}

Portions of this document may be illegible in electronic image products. Images are produced from the best available original document. 


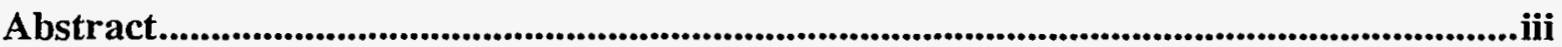

1. An Introduction to VCS Hints Displays ...............................................................................1

2. Hints Associated with VCS Panels ............................................................................................. 4

2.1: Page Description Panel .........................................................................................5

2.2: Data Selection and Data Selection Browser Panels ........................................... 7

2.3: Script I/O Panel.......................................................................................................... 8

2.4: CGM Panel ...................................................................................................... 10

2.5: Raster Panel................................................................................................................ 11

2.6: DRS Panel..............................................................................................................12

2.7: Indices Loop Panel.................................................................................................13

2.8: Print CGM Panel ...................................................................................................14

2.9: Colormap Editor/Table Panel...............................................................................16

2.10: Data Panel.......................................................................................................17

2.11: Graphics Method Panel..........................................................................................18

2.12: Template Panel...................................................................................................19

2.13: Template Browser Panel ......................................................................................20

2.14: (T) Line Table Panel ...........................................................................................21

2.15: (Tt) Text Table and (To) Text Orientation Table Panels...............................22

2.16: (Tm) Marker Table Panel .......................................................................................23

2.17: (Tf) Fillarea Table Panel ..............................................................................24

2.18: (Th) Format Table Panel.....................................................................................25

2.19: List Table Panel.......................................................................................................26

2.20: Compute Control Panel ...........................................................................................27

2.21: Animation Control Panel ...............................................................................28

2.22: Canvas .................................................................................................................30

2.23: Data Editor Panel.......................................................................................................31

2.24: Dimension Manipulation Panel ...........................................................................33

2.25: Dimension Assignment Panel.........................................................................35

2.26: Boxfill Editor Panel..........................................................................................36

2.27: Continents Editor Panel .......................................................................38

2.28: Isofill Editor Panel .................................................................................................39 
2.29: Isoline Editor Panel.....................................................................................................41

2.30: Outfill Editor Panel.........................................................................................................4

2.31: Outline Editor Panel ...........................................................................................44

2.32: Scatter Editor Panel..............................................................................................46

2.33: Vector Editor Panel ...........................................................................................4

2.34: Xvsy Editor Panel .............................................................................................49

2.35: Xyvsy Editor Panel .............................................................................................51

2.36: Yxvsx Editor Panel ...............................................................................................53

2.37: Line Editor Panel ..................................................................................................54

2.38: Text Editor Panel .........................................................................................................55

2.39: Marker Editor Panel..............................................................................................57

2.40: Fillarea Editor Panel..........................................................................................58

2.41: Format Editor Panel .......................................................................................60

2.42: List Editor Panel .................................................................................................661

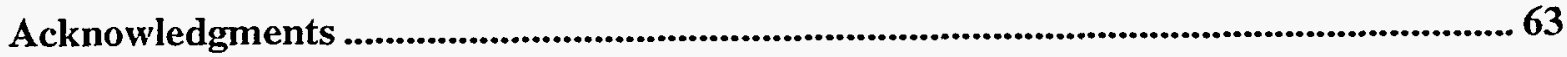




\section{ABSTRACT}

This report documents the Hints interactive displays that assist a user of the PCMDI Visualization and Computation System (VCS). These Hints describe the purpose of the VCS panels and provide basic instructions on their use. 


\section{An Introduction to VCS Hints Displays}

When any panel is accessed within the Visualization and Computation System (VCS), a brief description of its purpose and basic instructions on its use are provided in the form of Hints displays. (Note: because the Hints displays cover other VCS panels, they must be moved aside as $V C S$ is operated. The more experienced user may therefore wish to turn off the Hints-see instructions below.)

This Introduction provides a cursory overview of VCS, with an emphasis on how to select VCS panels from the Main Menu (see below). More detailed descriptions of the functions of VCS panels and instructions on their use are provided by associated Hints that are displayed as each VCS panel is selected. The VCS panels with associated Hints are described in Section 2. (The collection of Hints displays is also available as a postscript file for printing.)

\section{Main Menu}

Main Menu (Compact View)

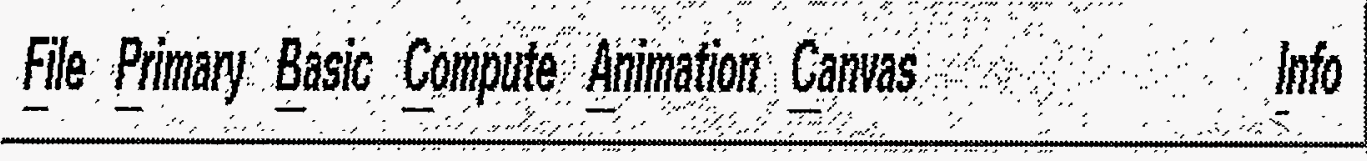

Main Menu (Full View)

\begin{tabular}{|c|c|c|c|c|c|c|c|c|}
\hline Mainkenul & SelectDáta & Scriptlo & SâvecGla! & SaveRas & SaveDRS & licés. & PrintCGM & Colormap \\
\hline Data & Boxill & Continents & Isofll & Isoline & Outfill & Outline & stal & Vector \\
\hline XusY & Xwsy & Yxusx & fertigne & Line & Text & 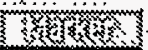 & 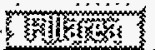 & Format \\
\hline List & Compute & Animation & Portrait] & Fullscreen & HintsOn & Examples & Authors & ExitvCS \\
\hline
\end{tabular}

Most VCS panels may be accessed via the Main Menu that is located at the top left of the screen above the VCS Message Panel. (Display of a VCS panel is terminated by selecting the 'Skull and Crossbones' button in the upper left corner of the panel.) The Main Menu may be displayed either in compact or full view (see figures above).

\section{How to Use the Main Menu}

Displaying Main Menu in Compact vs Full View:

To display the Main Menu in full view, select with the left mouse button the 'View All Menu Items' from the 'File' category of the compact Main Menu (via Main Menu - 'File' -> 'View All Menu Items'). To initiate VCS with the Main Menu in full view, save the state of a VCS session with the Main Menu in full view as a new initial.attributes file (included with installation of VCS)--see Hints on the Script I/O Panel (accessed via Main Menu - 'File' -> 'Script I/O') for procedures. To revert to compact view, select the 'Main Menu' button in the upper left corner of the 
full-view display. Note: Hints instructions for use of VCS panels assume the Main Menu is displayed in compact rather than full view.

Turning Off Hints:

When 'Hints On' is specified (via Main Menu - 'Info' -> 'Hints On'), selection of a VCS panel causes display of associated Hints. Turn off these Hints via Main Menu - 'Info'. - $\rightarrow$ 'Hints Off'. To initiate VCS without displaying Hints, save the state of a VCS session with 'Hints Off' as an initial.attributes file--see Hints associated with the panel accessed via Main Menu - 'File' -> 'Script I/O' for procedures.

\section{Selecting Primary Elements:}

In VCS, a graphic display page is made up of one or more pictures, each of which is defined by three primary elements--template, graphics method, and data. The template defines where to display, the graphics method defines how to display, and the data defines what to display. The template is selected by means of a Template Panel (accessed via Main Menu - 'Primary' -> 'Template Table'). The graphics method is selected by means of a Graphics Method Panel (accessed via Main Menu - 'Primary' -> 'Graphics Table'). The data is selected by means of a Data Panel (accessed via Main Menu - 'Primary' $\rightarrow$ 'Data Table'). See Hints associated with these VCS panels for details.

Selecting Basic Elements:

In VCS, the three primary elements (template, graphics method, data) are comprised of attribute sets that are defined by eight basic elements. These basic elements include the colormap (accessed via Main Menu - 'Basic' -> 'Colormap Editor/Table'); the line(accessed via Main Menu - 'Basic' -> 'Line Table (Tl)'); the text and text orientation (accessed via Main Menu - 'Basic' -> 'Text Table (Tt) and (To)'); the marker (accessed via Main Menu - 'Basic' -> 'Marker Table (Tm)'); the fillarea (accessed via Main Menu - 'Basic' -> 'Fillarea Table (Tf)'); the format (accessed via Main Menu 'Basic' $\rightarrow$ 'Format Table'); and the list (accessed via Main Menu - 'Basic' $\rightarrow$ 'List Table'). See Hints associated with each of these VCS panels for details.

\section{Plotting Data in VCS:}

Once the three primary elements (template, graphics method, data) are specified, a plot is displayed on the VCS Canvas--see associated Hints (accessed via Main Menu - 'Canvas' -> 'Portrait', 'Landscape', or 'Full Screen') for procedures.

\section{Animating Data in VCS:}

Data may be animated in VCS--see associated Hints (accessed via Main Menu - 'Animation' -> 'Animation CHints ontrol Panel') for procedures.

Computing in VCS:

Computations may be performed in VCS--see associated Hints (accessed via Main Menu 'Compute' $\rightarrow$ 'Compute Control Panel') for procedures. 
Saving DRS, Raster, and CGM Files:

A plot that is displayed on the VCS Canvas may be saved as a raster file, as a cgm file, or as a PCMDI Data Retrieval and Storage (DRS) file--see associated Hints (accessed via Main Menu 'File' -> 'Save DRS', 'Save Raster', and 'Save CGM') for procedures. Multiple sets of DRS, raster, or cgm output may also be saved to a single file--see associated Hints (accessed via Main Menu - 'File' -> 'Multi-Save (Indices)' ) for procedures.

Printing a CGM File:

A CGM file may be printed--see associated Hints (accessed via Main Menu - 'File' -> 'Print CGM') for procedures.

Saving a Script:

The state of a VCS session at any time may be saved (and later recovered) as a script--see associated Hints (accessed via Main Menu - 'File' -> 'Script I/O') for procedures. 


\section{Hints Associated with VCS Panels}

Hints associated with the following VCS panels are provided to assist the user. These Hints may be displayed interactively (via Main Menu - 'Info' -> 'Hints On') as each VCS panel is selected.

Page Description

Data Selection and Data Selection Browser

Script I/O

CGM

Raster

DRS

Indices Loop

Print CGM

Colormap Editor/Table

Data

Graphics Method

Template

Template Browser

(TI) Line Table

(Tt) Text Table and (To) Text Orientation Table

(Tm) Marker Table

(Tf) Fillarea Table

(Th) Format Table

List Table

Compute Control

Animation Control

Canvas

Data Editor

Dimension Manipulation

Dimension Assignment

Boxfill Editor

Continents Editor

Isofill Editor

Isoline Editor

Outfill Editor

Outline Editor

Scatter Editor

Vector Editor

XvsY Editor

Xyvsy Editor

Yxvsx Editor

Line Editor

Text Editor

Marker Editor

Fillarea Editor

Format Editor

List Editor 


\subsection{Page Description Panel}

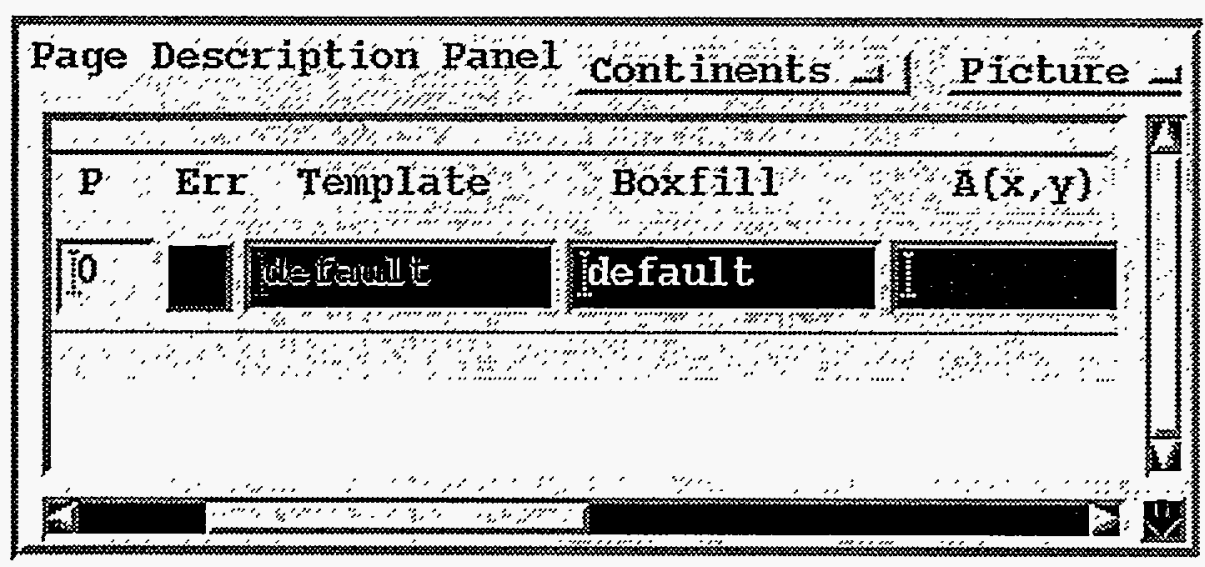

The Page Description Panel is used to specify picture descriptor forms that define how plots are displayed on the VCS Canvas (drawing area). There are three primary elements in each picture descriptor form that correspond to text windows of the template (in green), the graphics method (in blue), and the data (in red). Names are assigned to these text windows from the scroll windows below the Page Description Panel. These scroll windows include a green 'Template' window, a blue 'Graphics Method' window, and a red 'Data' window. (See Hints on the the Template Panel, the Graphics Method Panel; and the Data Panel for details.) Once template, graphics method, and data have been assigned to the picture descriptor form, the plot is drawn on the the VCS Canvas (see associated Hints).

\section{How to Use the Page Description Panel}

\section{Creating a Picture Descriptor Form:}

To create a new picture descriptor form, move the pointer over the 'Picture' menu button and hold down the left mouse button. Then move the pointer over 'Create' and release the left mouse button. A new picture descriptor form will appear at the end of the list.

\section{Removing a Picture Descriptor Form:}

Picture descriptor forms consist of two rows: a label row, including 'Id', 'P', 'On/Off/Err', Template', 'Graphics', 'Data'; and an input row, including text windows and a button. For quick removal of a picture descriptor form, move the pointer over the label row and press the right mouse button. As an alternative, select the label row with the left mouse button. Then move the pointer over the 'Picture' menu button and select the red 'Remove' menu item. All selected picture descriptor forms will be removed.

\section{Copying and Dropping a Template Name:}

Move the pointer to the green 'Template' scroll window below the Page Description Panel. To copy a template name, press and release the middle mouse button. To drop, move the pointer to the green 'Template' input text window of the desired page descriptor form, and press and release the middle mouse button. The selected template name will appear in the 'Template' input text window. 
Copying and Dropping a Graphics Method Name:

Move the pointer to the blue 'Graphics Method' scroll window below. To copy a graphics method name, press and release the middle mouse button. To drop, move the pointer to the blue 'Graphics' input text window of the desired page descriptor form, and press and release the middle mouse button. The selected graphics method name will appear in the 'Graphics Method' input text window.

\section{Copying and Dropping a Data Name:}

Move the pointer to the red 'Data' scroll window below. To copy a data name, press and release the middle mouse button. To drop, move the pointer to the red 'Data' input text window of the desired page descriptor form, and press and release the middle mouse button. The selected data name will appear in the 'Data' input text window.

\section{Monitoring the Status of a Picture Descriptor Form:}

The 'Status' button both controls and indicates the status of a picture. Before the picture descriptor form is created, the 'Status' button is red (indicating 'Error' status). When assignments are made (without error) to the 'Template', 'Graphics', and 'Data' input text windows, the button turns green (indicating 'On' status). Once the picture descriptor form is complete, toggling between 'On' (green) and 'Off' (yellow) states switches the display of the plot (in the VCS Canvas) on or off.

Showing the 'Id' of a Picture Descriptor Form:

Move the bottom horizontal scrollbar to the left. This action will uncover the picture descriptor form 'Id' name.

Setting the Picture Priority:

The picture with the highest priority number is displayed on top of all others. To change a priority, select the ' $\mathrm{P}$ ' input text window and enter the desired priority number, followed by a 'Return' or 'Enter'.

\section{Enlarging the Page Description Panel:}

Move the pointer to the green down arrow button in the lower right corner of the panel. Press and release the left mouse button. The panel will extend to the bottom of the VCS window. Select the green up arrow button to restore the panel to its former size.

\section{Drawing Continents:}

If the data to be plotted has longitude as the first dimension and latitude as the second dimension, and if 'Continents' is displayed on the Page Description Panel menu button, then continents will be drawn on the plot. If 'None' is displayed, then continents will not be be drawn. 


\subsection{Data Selection and Data Selection Browser Panels}

Access: via Main Menu - 'File' -> 'Select Data'

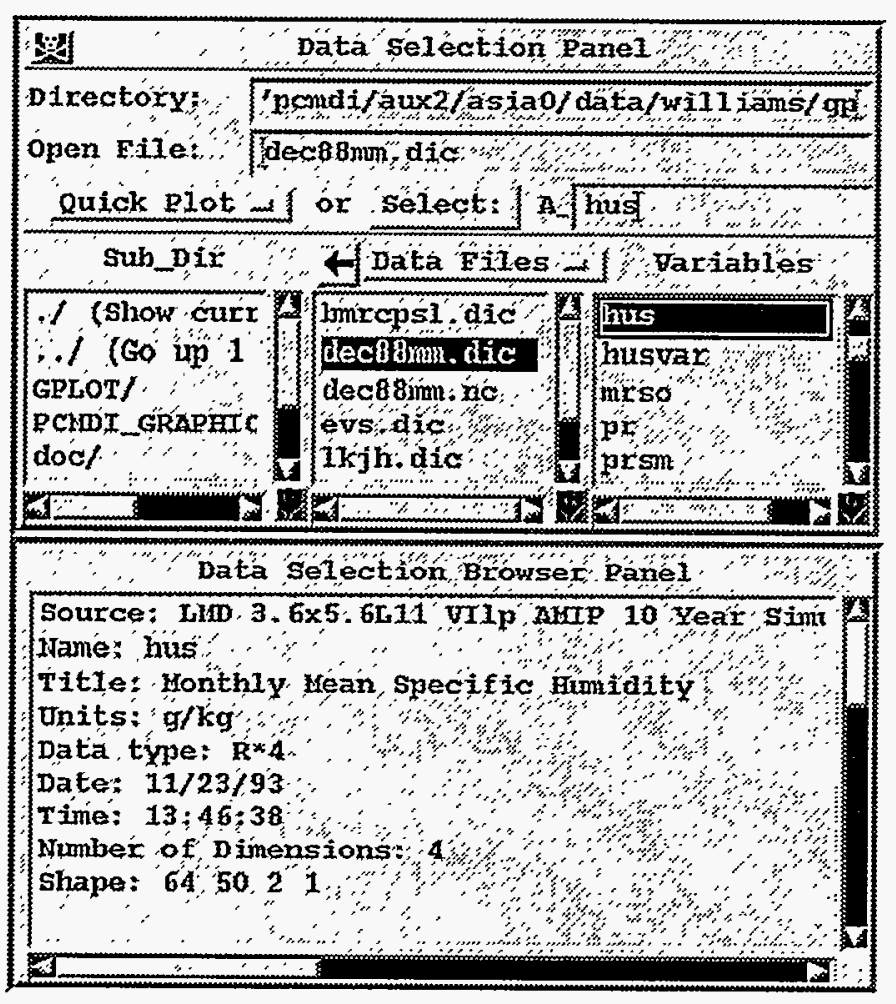

The Data Selection Panel is used for selecting data. The Data Selection Browser Panel displays the attributes of a data variable, including dimension information.

\section{How to Use The Data Selection and Data Selection Browser Panels}

\section{Changing Directories:}

Type the desired directory in the 'Directory' input text window. Press the 'Return' or 'Enter' key to register the end of typing. The 'Sub_Dir' scroll window and possibly also the 'Data Files' scroll window will change. (If the specified directory does not exist, the current directory will reappear.) The directory can also be changed by moving the pointer over a directory name in the 'Sub_Dir' scroll window and pressing the left mouse button.

\section{Selecting a Data File:}

Move the pointer over the desired data file name in the 'Data File' scroll window and press the left mouse button. A list of variable names will appear in the 'Variables' scroll window.

\section{Viewing a Variable:}

Move the pointer over a data variable name and press the left mouse button. Information showing the data variable's attributes (including dimension information) will appear below in the 'Data Selection Browser Panel'. 
Quick Plotting a Data Variable:

Move the pointer over the desired data variable name and press the left mouse button. Then move the pointer over the 'Quick Plot' button and press and hold the left mouse button. An assortment of graphics methods will appear. Select a graphics method by moving the pointer over the desired method and releasing the left mouse button. A plot of the data variable will appear on the VCS Canvas to the left.

Placing Data Variables in the Data Panel:

Move the pointer over a data variable name and double-press the left mouse button. The data variable name will appear in the red 'Data' scroll window above.

Changing the Name of the Selected Data Variable:

Move the pointer over the data variable name and press the left mouse button. The variable name will appear above in the 'A_' input text window. Modify the name and press the 'Select:' button to the left. The new data variable name will appear in the red 'Data' scroll window above.

\subsection{Script I/O Panel}

Access: via Main Menu - 'File' -> 'Script I/O'

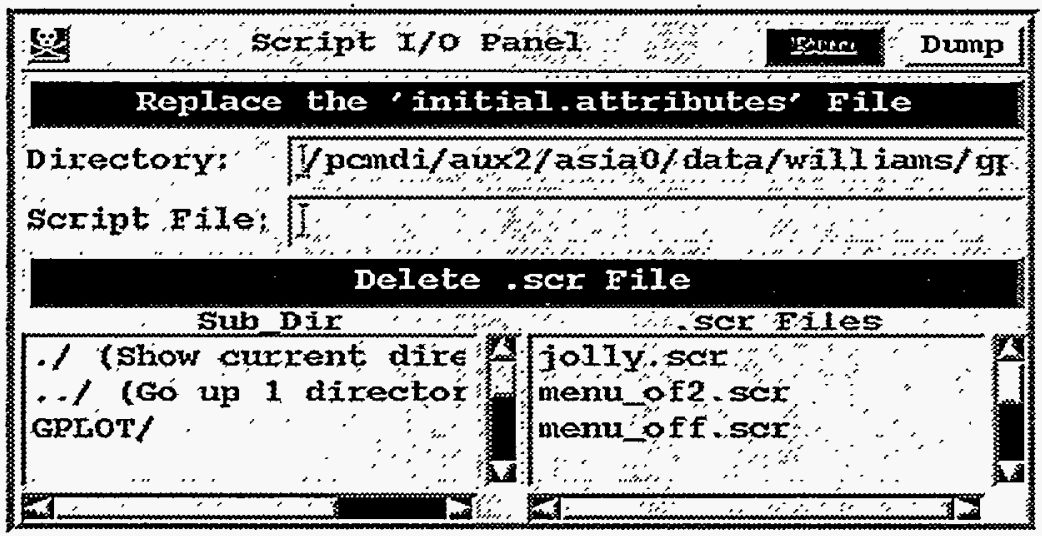

The Script I/O Panel is used to save the current state of VCS or to bring a previous state into VCS. It can also be used to replace the 'initial.attributes' file.

\section{How to Use the Script I/O Panel}

Replacing the 'initial.attributes' File:

Move the pointer over the blue 'Replace the initial.attributes File' button, then press the left mouse button. The 'initial.attributes' file, located in the 'PCMDI_GRAPHICS' directory, will be updated with the current VCS settings. The previous settings of the 'initial.attributes' file will be saved in file 'initial.attributes\%'. 
Changing the Script Directory:

Type the desired directory in the 'Directory' input text window. Press the 'Return' or 'Enter' key to register the end of typing. The 'Sub_Dir' scroll window and possibly the '.scr Files' scroll window will change. (If the specified directory does not exist, the current directory will reappear.) The directory can also be changed by moving the pointer over a directory name in the 'Sub_Dir' scroll window and pressing the left mouse button.

Selecting a Script File:

Move the pointer over the desired script file name in the '.scr File' scroll window and press the left mouse button. The 'Script File' input text window will display the selected script file.

Deselecting a Script File:

Move the pointer over the desired script file name in the '.scr File' scroll window and press the left mouse button. Then move the pointer over the red 'Delete Script File' button, and press the left mouse button.

Running a Script File:

Move the pointer over the desired script file name in the '.scr File' scroll window and press the left mouse button. Then move the pointer over the green 'Run' button, and press the left mouse button.

Saving or Dumping a Script File:

Move the pointer over the desired script file name in the '.scr File' scroll window and press the left mouse button, or enter a script name in the 'Script File:' input text window. Then move the pointer over the yellow 'Dump' button, and press the left mouse button. 


\subsection{CGM Panel}

Access: via Main Menu - 'File' -> 'Save CGM'

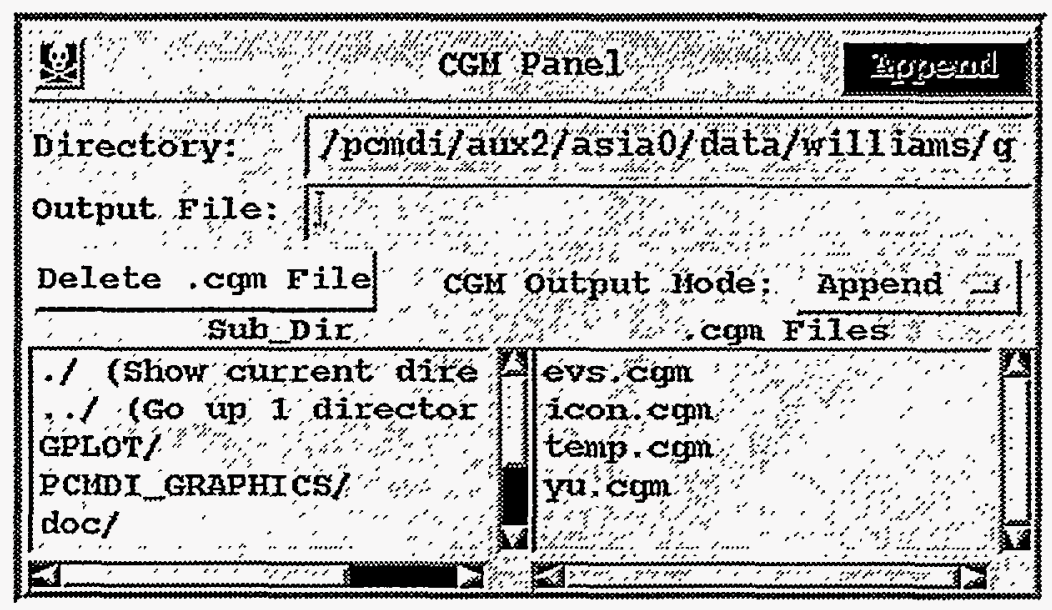

The CGM Panel is used to save or delete a cgm file. There are two modes for saving a cgm file: 'Append' mode appends cgm output to an existing cgm file; 'Replace' mode overwrites an existing cgm file with new cgm output.

\section{How to Use the CGM Panel}

\section{Changing the CGM Directory:}

Type the desired directory in the 'Directory' input text window. Press the 'Return' or 'Enter' key to register the end of typing. The 'Sub_Dir' scroll window and possibly also the '.cgm Files' scroll window will change. (If the specified directory does not exist, the current directory will reappear. The directory can also be changed by moving the pointer over a directory name in the 'Sub_Dir' scroll window and pressing the left mouse button.

\section{Selecting a CGM File:}

Move the pointer over the desired cgm file name in the '.cgm File' scroll window and press the left mouse button. The 'Output File' input text window will display the selected cgm file.

\section{Deleting a CGM File:}

Move the pointer over the desired CGM file name in the '.cgm File' scroll window and press the left mouse button. Then move the pointer over the red 'Delete CGM File' button, and press the left mouse button.

\section{Changing the CGM Output Mode:}

Move the pointer over the 'CGM Output Mode:' button, and press and hold the left mouse button. Move the pointer over 'Append' or 'Replace' and release the left mouse button. The green button in the upper right corner will change, showing the toggle selection. 


\subsection{Raster Panel}

Access: via Main Menu - 'File' -> 'Save Raster'

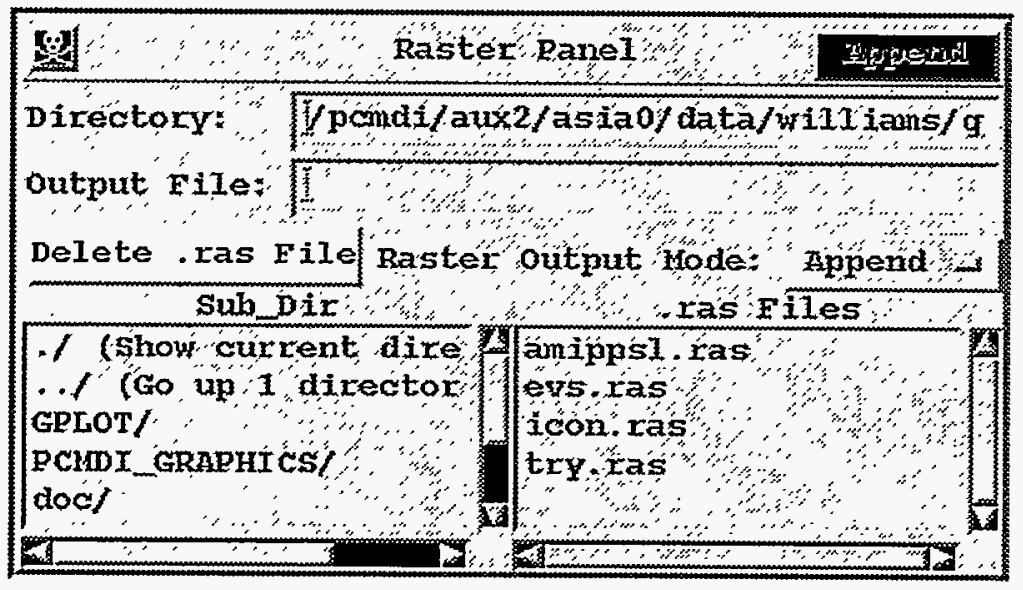

The Raster Panel is used to save or delete raster files. There are two modes for saving a raster file: 'Append' mode appends raster output to an existing raster file; "Replace' mode overwrites an existing raster file with new raster output.

\section{How to Use the Raster Panel}

Changing the Raster Directory:

Type the desired directory in the 'Directory' input text window. Press the 'Return' or 'Enter' key to register the end of typing. The 'Sub_Dir' scroll window and possibly also the 'ras Files' scroll window will change. (If the directory does not exist, the current directory will reappear.) The directory can also be changed by moving the pointer over a directory name in the 'Sub_Dir' scroll window and pressing the left mouse button.

\section{Selecting a Raster File:}

Move the pointer over the desired raster file name in the 'ras File' scroll window and press the left mouse button. The 'Output File' input text window will display the selected raster file.

\section{Deleting a Raster File:}

Move the pointer over the desired Raster file name in the '.ras File' scroll window and press the left mouse button. Now move the pointer over the red 'Delete ras File' button, and press the left mouse button.

\section{Changing the Raster Output Mode:}

Move the pointer over the 'Raster Output Mode:' button, and press and hold the left mouse button. Move the pointer over 'Append' or 'Replace' and release the left mouse button. The green button in the upper right corner of the Raster Panel will change, showing the toggle selection. 


\subsection{DRS Panel}

Access: via Main Menu - 'File' -> 'Save DRS'

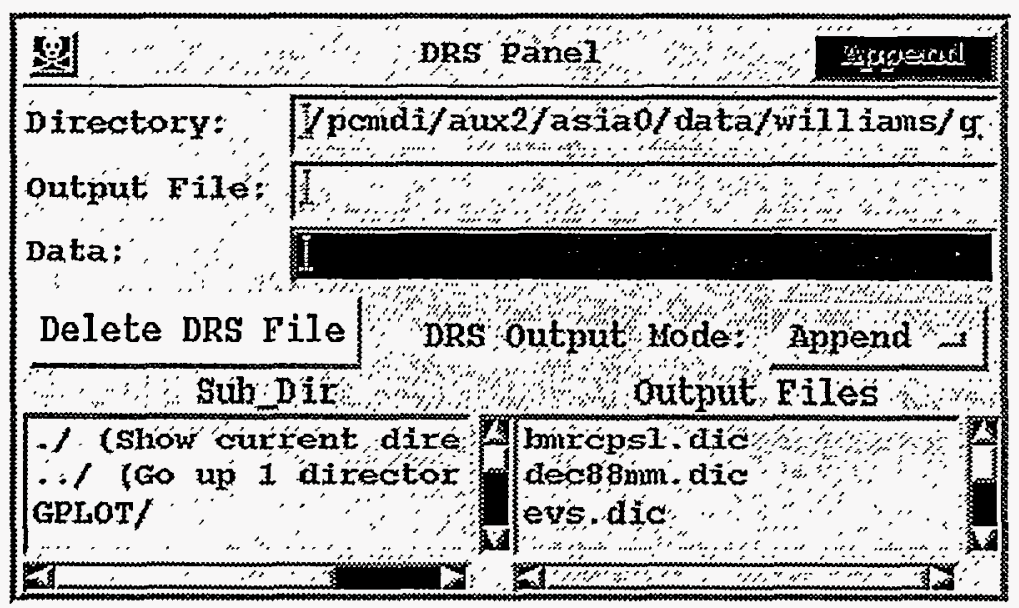

The DRS Panel is used to save or delete files that are written in the PCMDI Data Retrieval and Storage (DRS) format. There are two modes for saving a DRS file: 'Append' mode appends DRS output to an existing DRS file; 'Replace' mode overwrites an existing DRS file with new DRS output.

\section{How to Use the DRS Panel}

\section{Changing the DRS Directory:}

Type the desired directory in the 'Directory' input text window. Press the 'Return' or 'Enter' key to register the end of typing. The 'Sub_Dir' scroll window and possibly also the 'DRS Files' scroll window will change. (If the directory does not exist, the current directory will reappear.) The directory can also be changed by moving the pointer over a directory name in the 'Sub_Dir' scroll window and pressing the left mouse button.

\section{Selecting a DRS File:}

Move the pointer over the desired DRS file name in the '. dic File' scroll window and press the left mouse button. The 'Output File' input text window will display the selected DRS file.

\section{Deleting a DRS File:}

Move the pointer over the desired DRS file name in the 'DRS File' scroll window and press the left mouse button. Then move the pointer over the red 'Delete DRS File' button, and press the left mouse button.

\section{Changing the DRS Output Mode:}

Move the pointer over the 'DRS Output Mode:' button, and press and hold the left mouse button. Move the pointer over 'Append' or 'Replace' and release the left mouse button. The green button in the upper right corner of the DRS Panel will change, showing the toggle selection. 


\section{Selecting DRS Data:}

Move the pointer over the data name in the 'Data' scroll window and press the left mouse button. The data name will appear in the 'Data' input text window.

\subsection{Indices Loop Panel}

Access: via Main Menu - 'File' -> 'Multi-Save (Indices)'

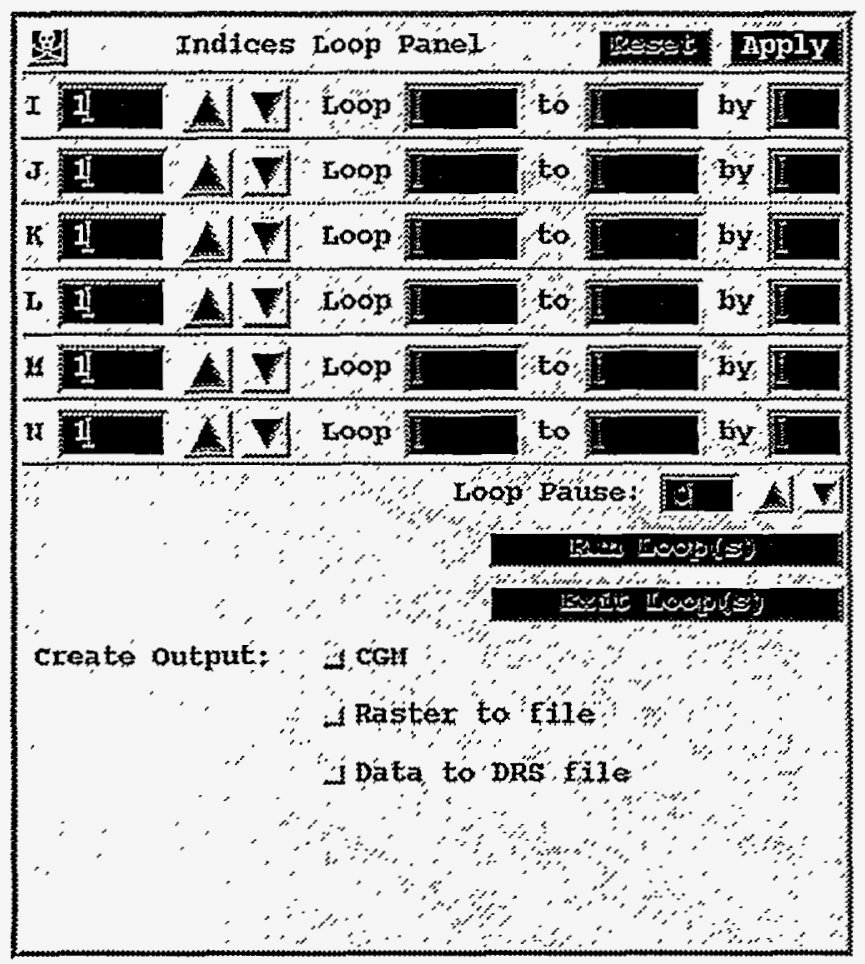

The Indices Loop Panel is used to loop through global indices (I, J, K, L, M, and N). It is also used to save multiple sets of cgm output, raster output, or DRS output in a single file.

\section{How to Use the Indices Loop Panel}

\section{Incrementing or Decrementing an Index:}

Values of the global indices ( $, J, K, L, M$, and $N$ ) are displayed in rows at the left side of the Indices Loop Panel along with corresponding up-arrows and down-arrows. To increment an index value, move the pointer over the corresponding up-arrow, and press the left mouse button. To decrement an index value, move the pointer over the corresponding down-arrow, and press the left mouse button. When finished incrementing or decrementing, press the red 'Apply' button to view changes.

\section{Looping On Indices:}

Looping on an index is implemented by specifying loop end points 'from' and 'to' with a 'by' step increment (e.g., ' $\mathrm{I}$ ' Loop from 1 to 10 by 1 ) in the green input text windows. The 'Loop' 
input text window receives the 'from' value, the 'to' window the 'to' value, and the 'by' window the step value. After the loop indices are set up, press the green 'Run Loop(s)' button to begin looping through the indices. Press the green 'Exit Loop(s)' button to stop looping. Press the 'Loop Pause' up-arrow or down-arrow to speed up or slow down viewing of the plots.

\section{Creating Output files:}

While looping on an index, cgm, raster, or DRS files can also be created. Press the 'CGM' toggle button to save cgm output. Press the 'Raster to file' toggle button to save raster output. Press the 'Data to DRS file' to save DRS output. The CGM Panel, Raster Panel, or DRS Panel will appear when the respective toggle buttons are selected (see associated Hints). To cancel output, reselect the toggle button(s).

\subsection{Print CGM Panel}

Access: via Main Menu - 'File' -> 'Print CGM'

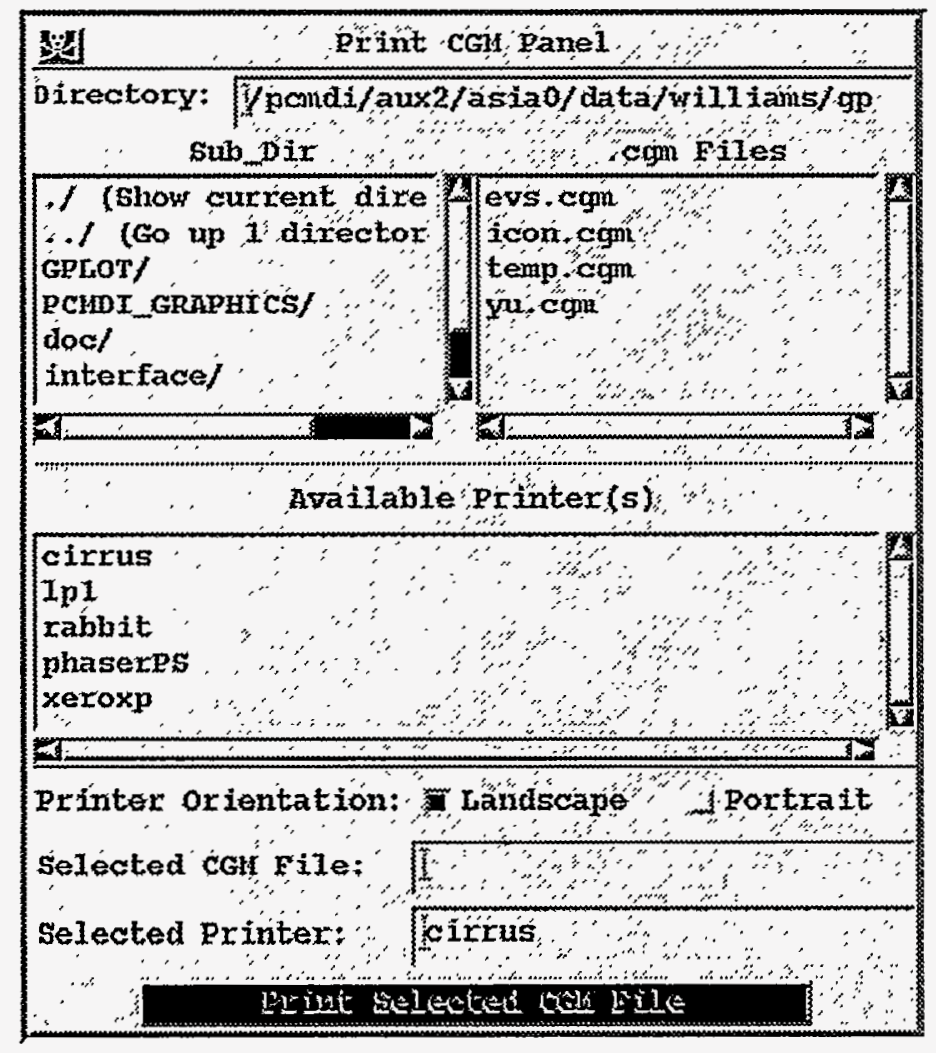

The Print CGM Panel is used to print CGM files out to a specified printer. The printer orientation mode is either specified as 'Landscape' (width exceeding height) or 'Portrait'(height exceeding width). 


\section{How to Use the Print CGM Panel}

Changing the Print CGM Directory:

Type the desired directory in the 'Directory' input text window. Press the 'Return' or 'Enter' key to register the end of typing. The 'Sub_Dir' scroll window and possibly also the '.cgm Files' scroll window will change. (If the directory does not exist, the current directory will reappear.) The directory can also be changed by moving the pointer over a directory name in the 'Sub_Dir' scroll window and pressing the left mouse button.

\section{Selecting a CGM File:}

Move the pointer over the desired cgm file name in the '.cgm File' scroll window and press the left mouse button. The 'Selected CGM File:' input text window will display the file name.

\section{Selecting an Output Printer:}

Move the pointer over the desired print device name in the 'Available Printer(s)' scroll window and press the left mouse button. The 'Selected Printer:' input text window (below) will display the chosen print device. Note: a list of available print devices is specified by the HARD_COPY file that is included with installation of VCS.

Changing the Print Orientation:

To specify landscape or portrait orientation in printing, select the corresponding 'Printer Orientation:' toggle button on the Print CGM Panel.

Sending CGM Files to the Designated Printer:

Select the green 'Print Selected CGM File' button by pressing the left mouse button. 


\subsection{Colormap Editor/Table Panel}

Access: via Main Menu - 'Basic' -> 'Colormap Editor/Table'

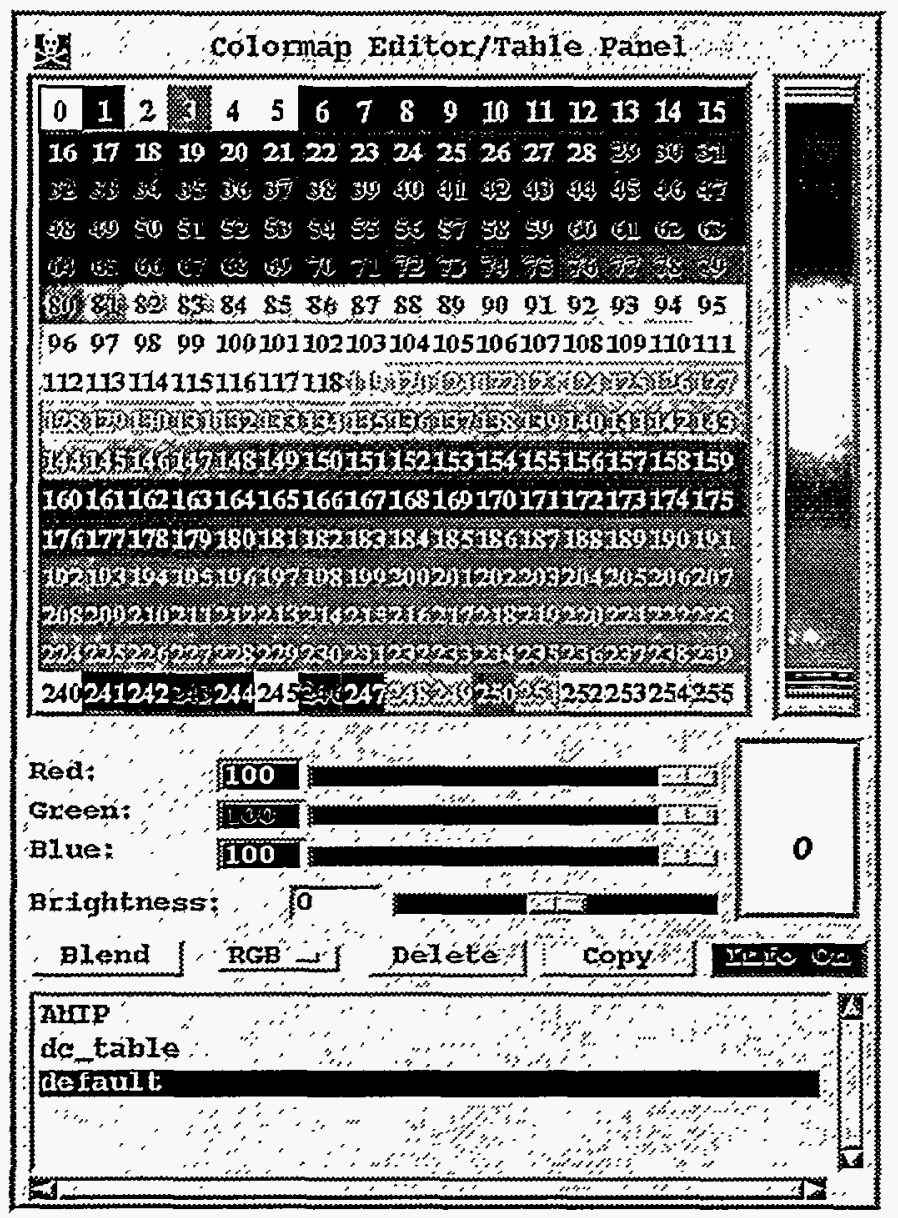

The Colormap Editor/Table Panel is used to create and modify colormaps. It contains 256 color indices, a color spectrum bar, RGB color sliders, a color index viewer and reset button, a brightness slider, a blend button, a color model menu button, a delete button, a copy button, an info button, and a scroll list of colormap names. VCS treats colormap attributes as one of the eight basic elements making up the three primary attribute sets (template, graphics method, and data). Note: A color index cannot be selected if the colormap is specified as 'default'or if the color index is greater than 240 .

\section{How to Use the Colormap Editor/Table Panel}

Selecting and Modifying a Color Index:

Move the pointer over one of the indices 0 through 239, and select with the left mouse button. The selected color index can then be modified with the 'Red', 'Green', and 'Blue' sliders. To reset the color index, select the button to the right of the corresponding slider. 


\section{Blending Colors:}

To blend colors, select a lower color index with the left mouse button and an upper color index with the middle mouse button, and modify the colors of each index as desired. Then press the 'Blend' button to change the colors of intermediate indices by progressively blending the colors of the lower and upper indices.

\section{Copying and Pasting Color Indices:}

To copy a range of color indices, select the first color index with the left mouse button, then select the second color index with the middle mouse button. To paste this range of color indices, move the pointer to the desired position, then press the right mouse button.

Copying/Renaming a Colormap:

Select a colormap name from the scroll window list, and press the 'Copy' button. An input text window will appear in which a new colormap name can be specified.

\section{Deleting a Colormap:}

Select a colormap name from the scroll window list, then press the 'Delete' button.

\section{Displaying Color Index Value Range Infomation:}

The green 'Info On' button will show color index value ranges only for the Boxfill graphics method. In that case, if the color index selected with the left or the middle mouse button is within the data range, then the VCS Message Panel (located below the Main Menu) will show a value range for the color index.

\subsection{Data Panel}

Access: via Main Menu - 'Primary' -> 'Data Table'

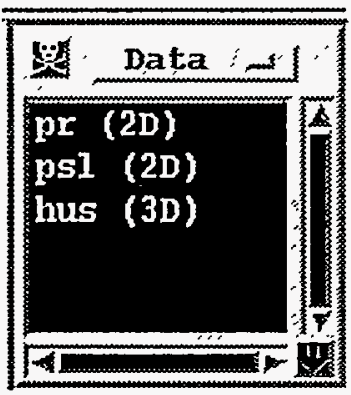

The Data Panel lists array data sets which are selected with the Data Selection Panel and are displayed on the Canvas (see associated Hints). VCS treats the data as one of the three primary element attribute sets, the others being the template and the graphics method. 


\section{How to use the Data Panel}

Selecting a Data Attribute Set:

Press and release the left mouse button on the desired data attribute set name. The Data Editor Panel will then appear below.

\section{Copying and Dropping a Data Attribute Set}

To copy a data attribute set, press and release the middle mouse button on the data attribute set name. To drop, move the pointer to the Page Description Panel (located above the Data Panel), and press and release the middle mouse button in the red text window.

Removing a Data Attribute Set:

Press and release the right mouse button on the data attribute set name to be removed.

\section{Using the 'Data' Menu Button:}

Pressing the 'Data' button displays a menu that allows the user to 'Select', 'Copy', 'Rename', or 'Remove' a data attribute set.

\subsection{Graphics Method Panel}

Access: via Main Menu - 'Primary' -> 'Graphics Table' -> 'Isoline', 'Outline', ‘Continents', 'Isofill', 'Outfill', 'Boxfill', 'Scatter', 'Vector', 'Xyvsy', 'XvsY', or 'Yxvsx'

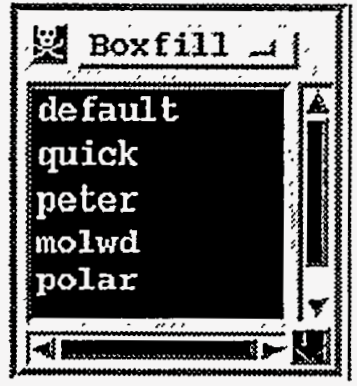

The Graphics Method Panel lists attribute sets corresponding to each of the available graphics methods which define how data are displayed on the Canvas: 'Boxfill', 'Continents', 'Isofill', 'Isoline', 'Outfill', 'Outline', 'Scatter', 'Vector', 'Xyvsy', 'XvsY', and 'Yxvsx'. VCS treats the graphics method as one of the three primary elements, the others being the template and the data. Note: the 'default' attribute sets cannot be removed or edited.

\section{How to use the Graphics Method Panel}

Selecting a Graphics Method Attribute Set:

Press and release the left mouse button on the desired attribute set name for the graphics method (selected via the Main Menu). An editor panel for the graphics method will appear below. 
Copying and Dropping a Graphics Method Attribute Set:

To copy, press and release the middle mouse button on a graphics method attribute set name. To drop, move the pointer to the Page Description Panel (located above the Graphics Method Panel), and press and release the middle mouse button in the blue text window.

Removing a Graphics Method Attribute Set:

Press and release the right mouse button on the graphics method attribute set name to be removed.

\section{Using the 'Graphics Method' Menu Button}

Pressing the button above the scroll window (whose label is the selected graphics method) displays a menu that allows the user to 'Copy', 'Rename', or 'Remove' a selected attribute set for the graphics method.

\subsection{Template Panel}

Access: via Main Menu - 'Primary' -> 'Template Table'

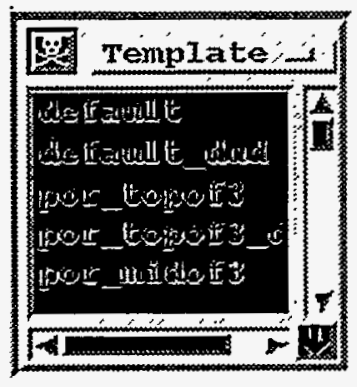

The template determines the location of each picture segment, the space to be allocated to it, and related properties relevant to its display. VCS treats the template as one of the three primary elements, the others being the graphics method and the data. Note: the 'default' template cannot be removed or edited.

\section{How to Use the Template Panel}

Selecting a Template Attribute Set:

Press and release the left mouse button on the desired template name. The Template Browser Panel (see associated Hints) will appear below .

Copying and Dropping a Template:

To copy a template, press and release the middle mouse button on the template name. To drop, move the pointer to the Page Description Panel (located above the Template Panel), and press and release the middle mouse button in the green input text window.

Removing a Template:

Press and release the right mouse button on the template name to be removed. 
Using the 'Template' Menu Button:

Pressing the 'Template' button displays a menu that allows the user to 'Copy', 'Rename', or 'Remove' a selected template attribute set.

\subsection{Template Browser Panel}

Access: via Template Panel -> Attribute Set Name
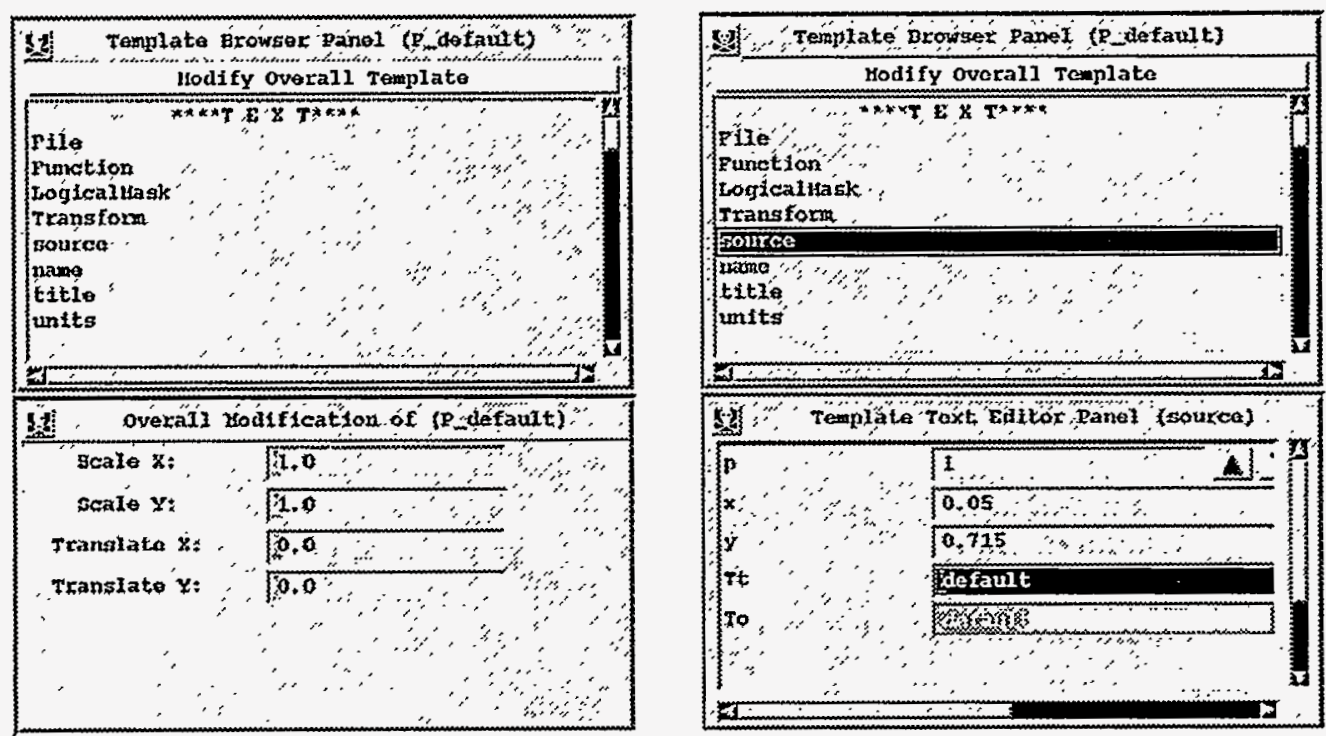

The Template Browser Panel lists subattributes of nine picture template categories: text, format, $\mathrm{x}$-tick marks, $\mathrm{y}$-tick marks, $\mathrm{x}$-labels, $\mathrm{y}$-labels, box/lines, legend space, and display space. These are designated in the Template Browser Panel scroll window with asterisks preceding and following each category name. The corresponding subattributes also appear below the category name in the scroll window.

\section{How to Use the Template Browser Panel}

\section{Modifying the Overall Template:}

All the subattributes can be scaled and translated at once by selecting the yellow 'Modify Overall Template' button. The subpanel labeled 'Overall Modification of (template name)' then will appear below the Template Browser Panel. Scale or translate the template by modifying the desired input text window. Press the red 'Apply' button to register changes.

\section{Modifying a Subattribute:}

Position the pointer over the desired subattribute name and press the left mouse button. A panel will appear below the Template Browser Panel to allow editing of the selected subattribute. Press the red 'Apply' button to register changes. 
Changing the Priority of a Subattribute:

To change the priority of a subattribute, enter a new integer value in the ' $p$ ' input text window of the subattribute's editing panel. The subattribute with the highest priority number is displayed on top of all others on the VCS Canvas, and a subattribute with priority 0 is not displayed. The ' $p$ ' input text window can also be changed by using the up-down arrow.

\section{Changing the Position of a Subattribute:}

The position of the subattribute can be determined by the input text windows labeled ' $x$ ', ' $y$ ', ' $x 1$ ', ' $y 1$ ', ' $x 2$ ', or ' $y 2$ '. By changing the values displayed in these windows, the subattribute can be moved to the desired position on the VCS Canvas.

\section{Changing a Basic Element of a Subattribute:}

If a subattribute uses one of the eight basic elements (e.g., line, marker, text, etc.), then the corresponding input text window is labeled (e.g., ' $\mathrm{Tl}$ ' for line, ' $\mathrm{Tm}$ ' for marker, ' $\mathrm{Tt}$ ' for text, etc.) and colored (e.g., cyan for line, brown for marker, magenta for text, etc.) accordingly. To change a basic element of a subattribute, access the appropriate basic element panel, and copy and drop the desired basic element attribute name in the input text window. (See Hints associated with the basic element panels for copy and drop procedures.)

\subsection{4 (TI) Line Table Panel}

Access: via Main Menu - 'Basic' -> 'Line Table (Tl)'

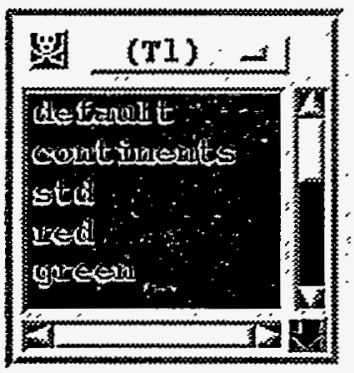

The (Tl) Line Table Panel contains a list of line attribute names that define the line type, width, and color index. VCS treats the line attribute as one of the eight basic elements that are used in describing the three primary elements (template, graphics method, data). Note: the 'default' line cannot be removed or edited.

\section{How to Use the (Tl) Line Table Panel}

Selecting Line:

Press and release the left mouse button on a line attribute set name. The Line Editor Panel (see associated Hints) will appear below. 
Copying and Dropping Line:

To copy line, press and release the middle mouse button on the desired line name. To drop, move the pointer to a text window slot that has the same cyan color as the (Tl) Table, and press and

release the middle mouse button in this slot.

Removing Line:

Press and release the right mouse button on the line attribute set name to be removed.

Using the '(Tl)' Menu Button:

Pressing the '(Tl)' button displays a menu that allows the user to 'Copy', 'Rename', or 'Remove' a selected line attribute set.

\subsection{5 (Tt) Text Table and (To) Text Orientation Table Panels}

Access: via Main Menu - 'Basic' -> 'Text Table (Tt) and (To)'

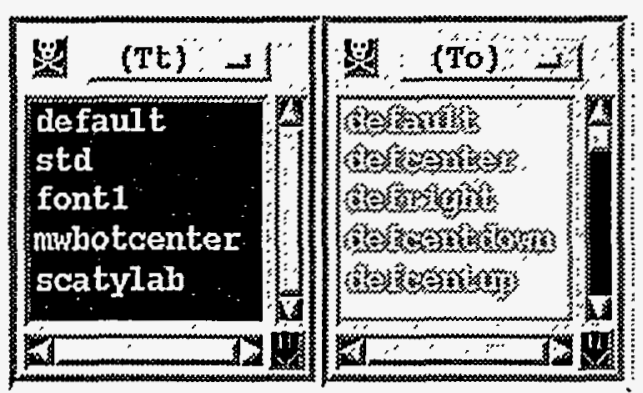

The (Tt) Text and (To) Text Orientation Tables are displayed simultaneously.

The (Tt) Text Table lists text attribute set names that define the font, spacing, expansion, and color index. VCS treats the text attribute as one of the eight basic elements that are used in describing the three primary elements (template, graphics method, data). Note: the 'default' text cannot be removed or edited.

The (To) Text Orientation Table lists text orientation attribute set names that define the character height, angle, path, and horizontal and vertical alignment. VCS treats the text orietation attribute as one of the eight basic elements that are used in describing the three primary elements (template, graphics method, data). Note: the 'default', 'defcenter', 'defright', 'defcentdown', and 'defcentup' text orientation cannot be removed or edited.

\section{How to Use the (Tt) Text and (To) Text Orientation Table Panels}

\section{Selecting Text or Text Orientation:}

Press and release the left mouse button on a text or text orientation attribute set name. The Text Editor Panel (see associated Hints) will appear below. 
Copying and Dropping Text:

To copy text, press and release the middle mouse button on the desired text attribute set name. To drop, move the pointer to a text window slot that has the same magenta color as the (Tt) Table, and press and release the middle mouse button in this slot.

Copying and Dropping Text Orientation:

To copy text orientation, press and release the middle mouse button on the desired text orientation attribute set name. To drop, move the pointer to a text orientation window slot that has the same orange color as the (To) table, and press and release with the middle mouse button in this slot.

Removing Text or Text Orientation:

Press and release the right mouse button on the text or text orientation attribute set name to be removed.

Using the '(Tt)' Menu Button:

Pressing the '(Tt)' button allows the user to 'Copy', 'Rename', or 'Remove' a selected text attribute set.

Using the '(To)' Menu Button:

Pressing the '(To)' button displays a menu that allows the user to 'Copy', 'Rename', or 'Remove' a selected text orientation attribute set.

\subsection{6 (Tm) Marker Table Panel}

Access: via Main Menu - 'Basic' -> 'Marker Table (Tm)'

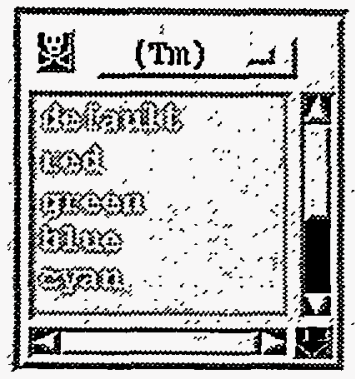

The (Tm) Marker Table Panel lists marker attribute set names that define the marker type, size, and color index. VCS treats the marker attribute as one of the eight basic elements that are used in describing the three primary elements (template, graphics method, data). Note: the 'default' marker cannot be removed or edited.

\section{How to Use the (Tm) Marker Table Panel}

Selecting a Marker:

Press and release the left mouse button on a marker attribute set name. The Marker Editor Panel (see associated Hints) will appear below. 
Copying and Dropping a Marker:

To copy a marker, press and release the middle mouse button on the desired marker attribute set name. To drop, move the pointer to a text window slot that has the same brown color as the (Tm) Table, and press and release the middle mouse button in this slot.

\section{Removing a Marker:}

Press and release the right mouse button on the marker attribute set name to be removed.

Using the '(Tm)' Menu Button:

Pressing the ' $(\mathrm{Tm})$ ' button displays a menu that allows the user to 'Copy', 'Rename', or 'Remove' a selected marker attribute set.

\subsection{7 (Tf) Fillarea Table Panel}

Access: via Main Menu - 'Basic' -> 'Fillarea Table (Tf)'

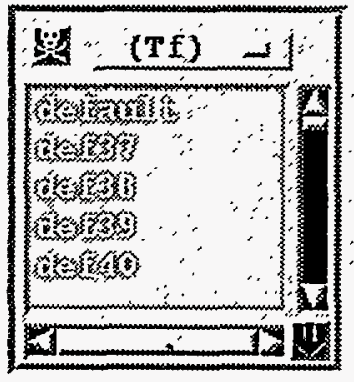

The (Tf) Fillarea Table Panel lists fillarea attribute set names that define the fillarea style, style index, and color index. VCS treats the fillarea attribute as one of the eight basic elements that are used in describing the three primary elements (template, graphics method, data). Note: the 'default' fillarea cannot be removed or edited.

\section{How to Use the (Tf) Fillarea Table Panel}

\section{Selecting Fillarea:}

Press and release the left mouse button on a fillarea attribute set name. The Fillarea Editor Panel (see associated Hints) will appear below.

\section{Copying and Dropping Fillarea:}

To copy fillarea, press and release the middle mouse button on the desired fillarea attribute set name. To drop, move the pointer to a text window slot that has the same olive green color as the (Tf) Table, and press and release the middle mouse button in this slot.

\section{Removing Fillarea:}

Press and release the right mouse button on the fillarea attribute set name to be removed. 
Using the '(Tf)' Menu Button:

Pressing the '(Tf)' button displays a menu that allows the user to 'Copy', 'Rename', or 'Remove' a selected fillarea attribute set.

\subsection{8 (Th) Format Table Panel}

Access: via Main Menu - 'Basic' -> 'Format Table (Th)'

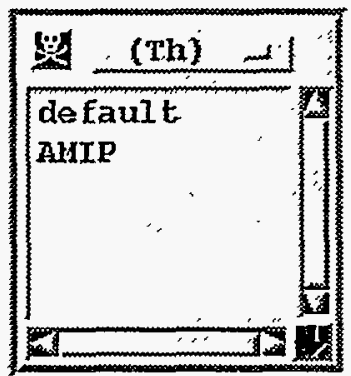

The (Th) Format Table Panel lists format attribute set names that define the format, name, and units of a dimension. VCS treats the format attribute as one of the eight basic elements that are used in describing the three primary elements (template, graphics method, data). Note: the 'default' format cannot be removed or edited.

\section{How to Use the (Th) Format Table Panel}

\section{Selecting a Format:}

Press and release the left mouse button on a format attribute set name. The Format Editor Panel (see associated Hints) will appear below.

Copying and Dropping a Format:

To copy format, press and release the middle mouse button on the desired format attribute set name. To drop, move the pointer to a text window slot that has the same light green color as the (Th) Table, and press and release the middle mouse button in this slot.

Removing a Format:

Press and release the right mouse button on the format attribute set name to be removed.

Using the '(Th)' Menu Button:

Pressing the '(Th)' button displays a menu that allows the user to 'Copy', 'Rename', or 'Remove' a selected format attribute set. 


\subsection{List Table Panel}

Access: via Main Menu - 'Basic' -> 'List Table'

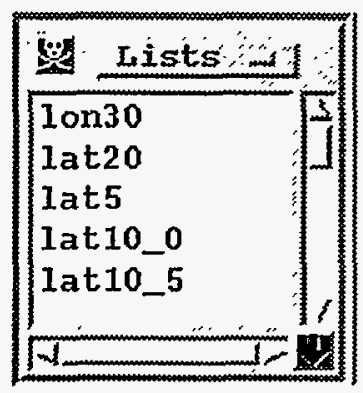

The List Table Panel contains list attribute set names that define sequential pairs of values and corresponding strings (e.g., longitude values and labels). VCS treats the list attribute as one of the eight basic elements that are used in describing the three primary elements (template, graphics method, data). Note: the 'lon30' or 'lat 20 ' lists cannot be removed or edited.

\section{How to Use the List Table Panel}

\section{Selecting a List:}

Press and release the left mouse button on a list attribute set name. The List Editor Panel (see associated Hints) will appear below.

\section{Copying and Dropping a List:}

To copy a list, press and release the middle mouse button on the desired list attribute set name. To drop, move the pointer to a text window slot that has the same yellow color as the List Table, and press and release the middle mouse button in this slot.

Removing a List:

Press and release the right mouse button on the list attribute set name to be removed.

\section{Using the 'Lists' Menu Button:}

Pressing the 'Lists' button displays a menu that allows the user to 'Copy', 'Rename', or 'Remove' a selected list attribute set. 


\subsection{Compute Control Panel}

Access: via Main Menu - 'Compute' -> 'Compute Control Panel'

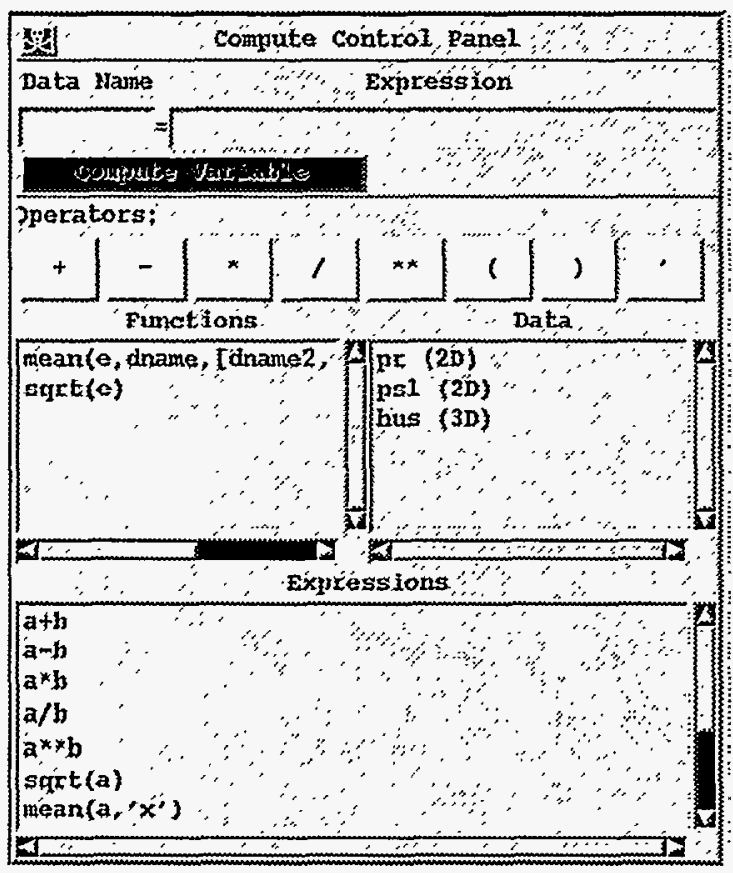

The Compute Control Panel is used to compute new data variables. In order to perform computations, data variables must be visible in the Data Panel (see Hints on the Data Panel and on the Data Selection Panel).

\section{How to Use the Compute Control Panel}

Specifying New Data Name and Expression:

Move the pointer into the 'Data Name' input text window and press the left mouse button. Enter the new data name. Then move the pointer into the 'Expression' input text window and press the left mouse button. Enter the desired expression. (The available 'Operators', 'Functions', 'Expressions' and 'Data' are listed. Selection of a particular operator, function, expression, or data will place it in the 'Expression' input text window.)

\section{Computing a Variable:}

Once the desired selections are visible in the 'Data Name' and 'Expression' input text windows (see above), press the green 'Compute Variable' button. The computed data variable name will be displayed in the red Data Panel (located above the Compute Control Panel), where a ' $c$ ' appended to the name confirms completion of the computation. 


\subsection{Animation Control Panel}

Access: via Main Menu - 'Animation' -> 'Animation Control Panel'

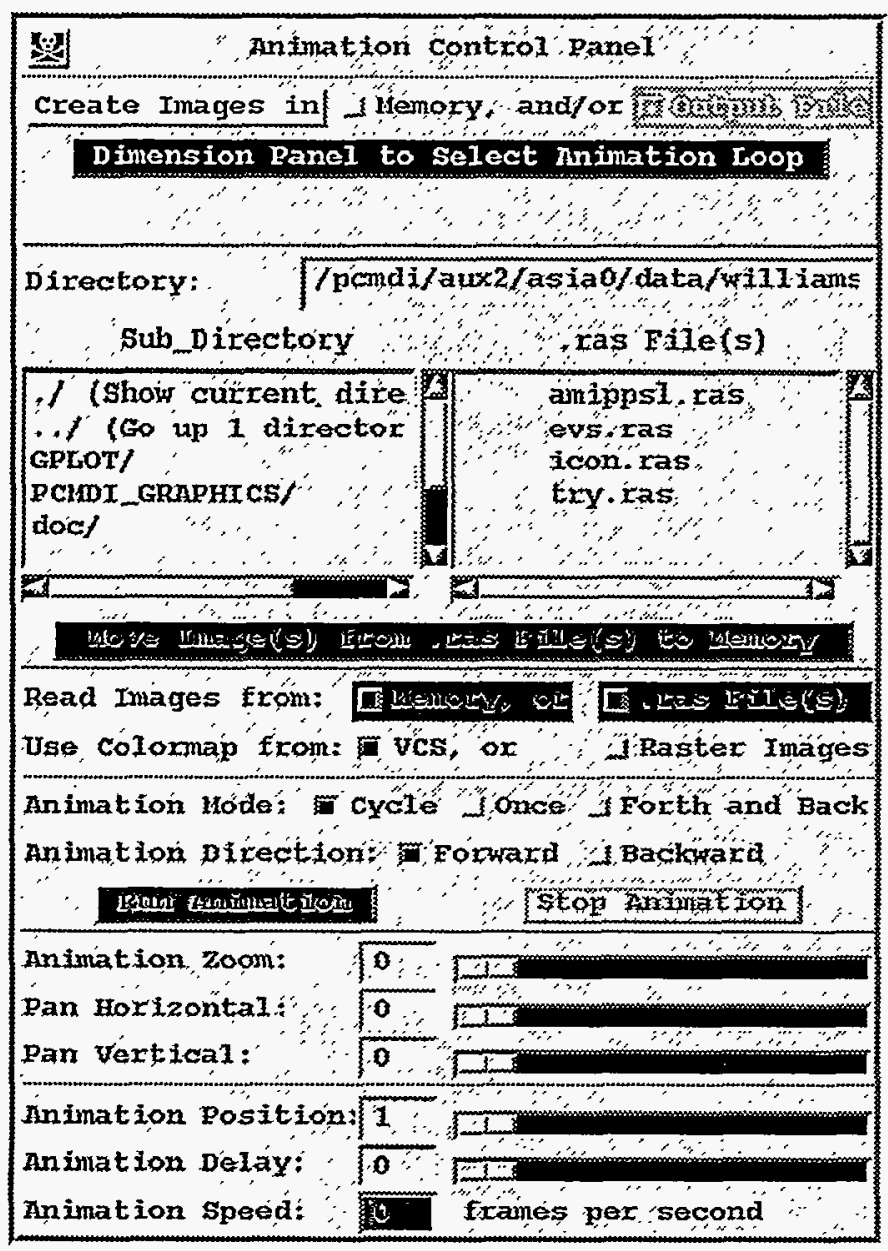

The Animation Control Panel is used to create sequences of raster images in memory or in an output file (i.e., 'filename.ras'). Once raster images are created, their animation can be viewed subject to user control.

\section{How to Use the Animation Control Panel}

Before the Animation Control Panel is accessed, there must be at least one plot of data having three or more dimensions displayed on the VCS Canvas. (An unlimited number of plots can be animated, but they all must use the same data variable. To create a raster file for animation of more than one data variable, see Hints on the Indices Panel and on the Data Editor Panel.) Once this condition is satisfied, use the Animation Control Panel to select whether to create images in 'Memory' and/or in an 'Output File'. If the 'Output File' toggle button is selected, an input text window will appear below to receive the output file name. Press the yellow 'Create Images in' button to begin saving raster images. When saving is completed, this button will turn red and display 'Stop Creating:'. Press the 'Stop Creating:' button to stop saving raster images at any time. 
Moving Raster Files into Memory:

In the '.ras File(s)' scroll window, select the raster file that is desired first in memory by moving the pointer over the raster file name and pressing the left mouse button. Then select raster files 2 , 3 , etc. in the same manner. The order of raster files in memory will be designated by a ' 1 ', ' 2 ', ' 3 ', etc. that is prepended to each raster file name. To remove a raster file from the sequence list, reselect the raster file name; the prepending number will be removed and the list will reorder itself. After selecting the desired files, press the green 'Move Image(s) from .ras File(s) to Memory' button to move the raster file images into memory.

\section{Reading Images from Memory or from Raster Files:}

If images are saved in memory or raster files images are moved into memory, the 'Read Images from:' toggle is automatically set to 'Memory'. To read images from raster files, select the files to be animated in the '.ras File(s)' scroll window, and set the 'Read Images from:' toggle to '.ras File(s)'.

\section{Setting the Animation Colormap:}

If the 'Use Colormap from:' toggle is set to 'VCS', then VCS controls the colormap. In this state, the Colormap Editor/Table Panel can be accessed (see associated Hints), and color indices can be modified while animation is proceeding. If instead the 'Use Colormap from:' toggle is set to 'Raster Images', then the colormap stored with each image is used.

\section{Setting the Animation Mode:}

If the 'Animation Mode:' is set to 'Cycle', then the sequence of images will be repeated regularly, as in a loop. If the 'Animation Mode:' is set to 'Once', then each image is displayed after animation stops. If the 'Animation Mode:' is set to 'Forth and Back', animation proceeds to the end of the sequence of images, and then reverses direction.

\section{Running or Stopping Animation:}

Once the images are loaded into memory or the raster files containing images are selected (see above), the user can select the 'Read Images from:' toggle to be either 'Memory' or '.ras File(s)'. Then press the cyan 'Run Animation' button to start animation. To stop animation, press the orange 'Stop Animation' button.

\section{Panning and Zooming Animation:}

The animation 'Pan' and 'Zoom' controls are not currently implemented in VCS.

\section{Controlling Animation Position, Delay, and Speed:}

The 'Animation Position' input text window shows the image frame that is being displayed. If animation is stopped, the 'Animation Position' slider can be moved to view a particular frame. The 'Animation Delay' slider slows the animation speed: the higher the number, the slower the speed. The 'Animation Speed' indicator shows the number of frames being viewed per second.

\section{Setting-Up the Dimensions for Animation Loop:}

To select the desired dimension values for animation, move the pointer over the blue Dimension Panel to the 'Select Animation Loop' button and press the left mouse button. The Dimension Manipulation Panel (see associated Hints) will appear, allowing the user to reverse, stride, or select dimension values. 
Changing the Animation Directory:

Type the desired directory in the 'Directory' input text window. Press the 'Return' or 'Enter' key to register the end of typing. The 'Sub_Dir' scroll window and possibly also the '.ras File(s)' scroll window will change. (If the specified directory does not exist, the current directory will reappear. ) The directory can also be changed by moving the pointer over a directory name in the 'Sub_Directory' scroll window and pressing the left mouse button.

\subsection{Canvas}

Access: via Main Menu - 'Canvas' -> 'Landscape', 'Canvas' -> 'Portrait', 'Canvas' -> 'Full Screen', or 'Canvas' $\rightarrow$ 'Image Zoom'

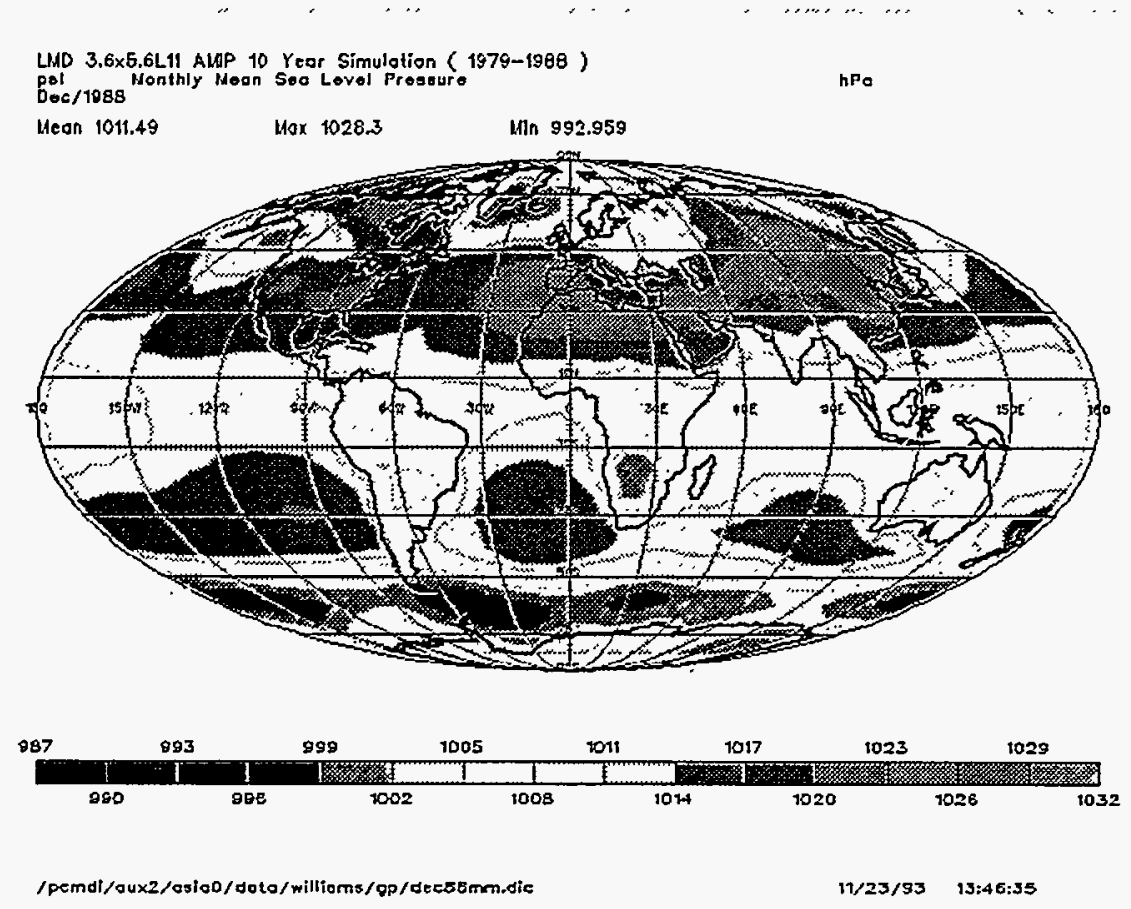

The Canvas is used to display plots and animations in VCS. It is always visible on the screen in a landscape (width exceeding height), portrait (height exceeding width), or full-screen mode.

\section{How to Use the Canvas}

\section{Designating Landscape Layout:}

To view the Canvas in landscape mode, move the pointer to the 'Canvas' pull down menu. Press and hold the left mouse button, then move the pointer over the 'Landscape' option and release. The Canvas layout will change accordingly.

\section{Designating Portrait Layout:}

To view the Canvas in portrait mode, move the pointer to the 'Canvas' pull down menu option. Press and hold the left mouse button, then move the pointer over the 'Portrait' option and release. The Canvas layout will change accordingly. 


\section{Designating Full-Screen Canvas:}

To view the Canvas in full-screen mode, move the pointer to the 'Canvas' pull down menu. Press and hold the left mouse button, then move the pointer over 'Full Screen' option and release. The Canvas will cover all VCS panels and buttons, with the exception of the red 'Restore Size' button, which can be selected to restore the Canvas to its former size.

\section{Zooming Canvas Image:}

To zoom in on an image that is displayed on the Canvas, move the pointer inside the Canvas and mark the image with the left mouse button. With the middle mouse button, hold down and move the pointer to the bottom of the image to be zoomed. A box outlining this image will be displayed. Release the middle mouse button to zoom in on the outlined box. Press the right mouse button to restore the original image.

\subsection{Data Editor Panel}

Access: via Data Panel -> Attribute Set Name

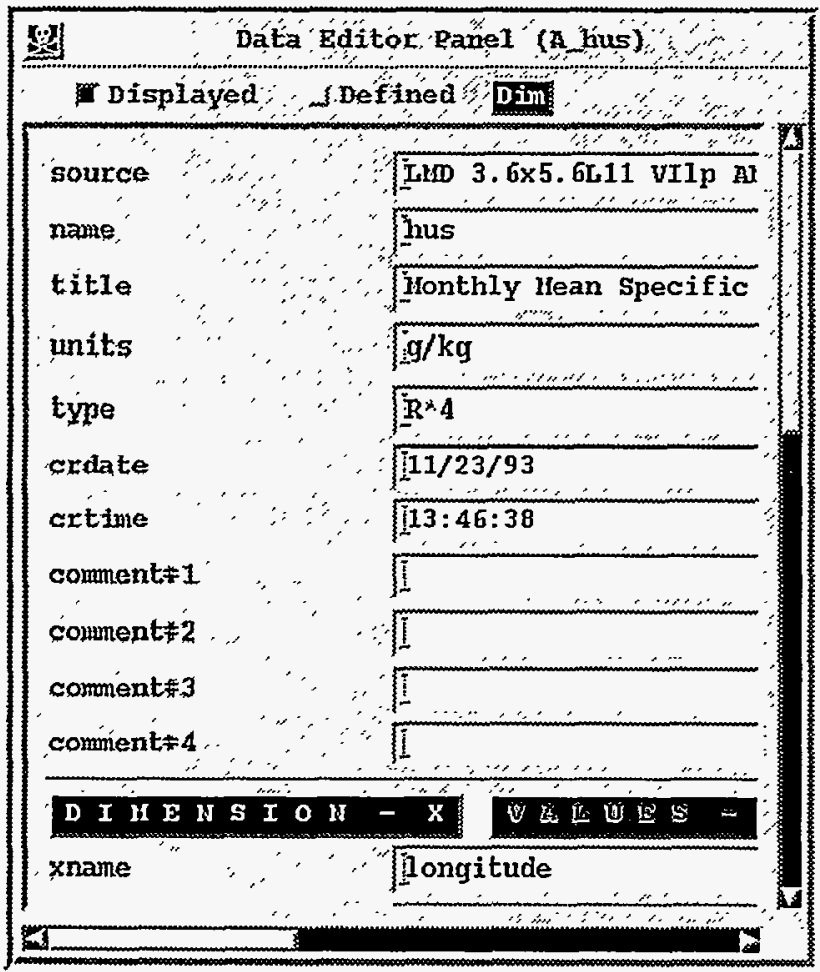

The Data Editor Panel lists a 'Displayed' and a 'Defined' array data attribute set. The 'Displayed' attribute set represents what will be displayed on the VCS Canvas, while the 'Defined' attribute set defines the data variable. 


\section{How to Use the Data Editor Panel}

Viewing the 'Displayed' or 'Defined' Data Attribute Set:

At the top of the Data Editor Panel there are two toggle buttons--'Displayed' and 'Defined'. Select 'Displayed' to view the displayed array data attribute set, or select 'Defined' to view the defined array data attribute set.

\section{Changing an Input Text Window:}

To change a yellow input text window, move the pointer into the window and press the left mouse button. If the text is modified, a green equal sign will appear, representing an assignment (i.e., 'source' = 'user-specified text statement'). When modifying an input text window, 'Reset' and 'Apply' buttons appear in the upper right corner. Select 'Reset' to bring the currently modified input text window back to its original statement. If the green equal sign is selected, the message '*******Apply to see New String******' will appear. Then, if the red 'Apply' button is selected, the defined data attribute set string will appear in the yellow input text window.

\section{Entering a List Name in an Input Text Window:}

Access the List Table Panel (via Main Menu - 'Basic' -> 'List Table') and move the pointer into the yellow 'Lists' scroll window. To copy a list, press and release the middle mouse button on the desired list attribute set name. To drop, move the pointer to the desired yellow input text window in the Data Editor Panel and press the middle mouse button. The selected list attribute set name will appear in the input text window.

Accessing the Dimension Manipulation Panel:

Select the blue 'Dim' button at the top of the Data Editor Panel to access the Dimension Manipulation Panel (see associated Hints). Then, to view the desired dimension column and coordinate values, select the blue 'DIMENSIONS- ?' button (where ? represents dimension X, Y, Z, etc.).

Accessing the Dimension Assignment Panel:

Select the cyan 'VALUES - ?' button (where, ? represents dimension X, Y, Z, etc.) to access the Dimension Assignment Panel (see associated Hints). 


\subsection{Dimension Manipulation Panel}

Access: via Data Editor Panel -> 'DIMENSION - ' button

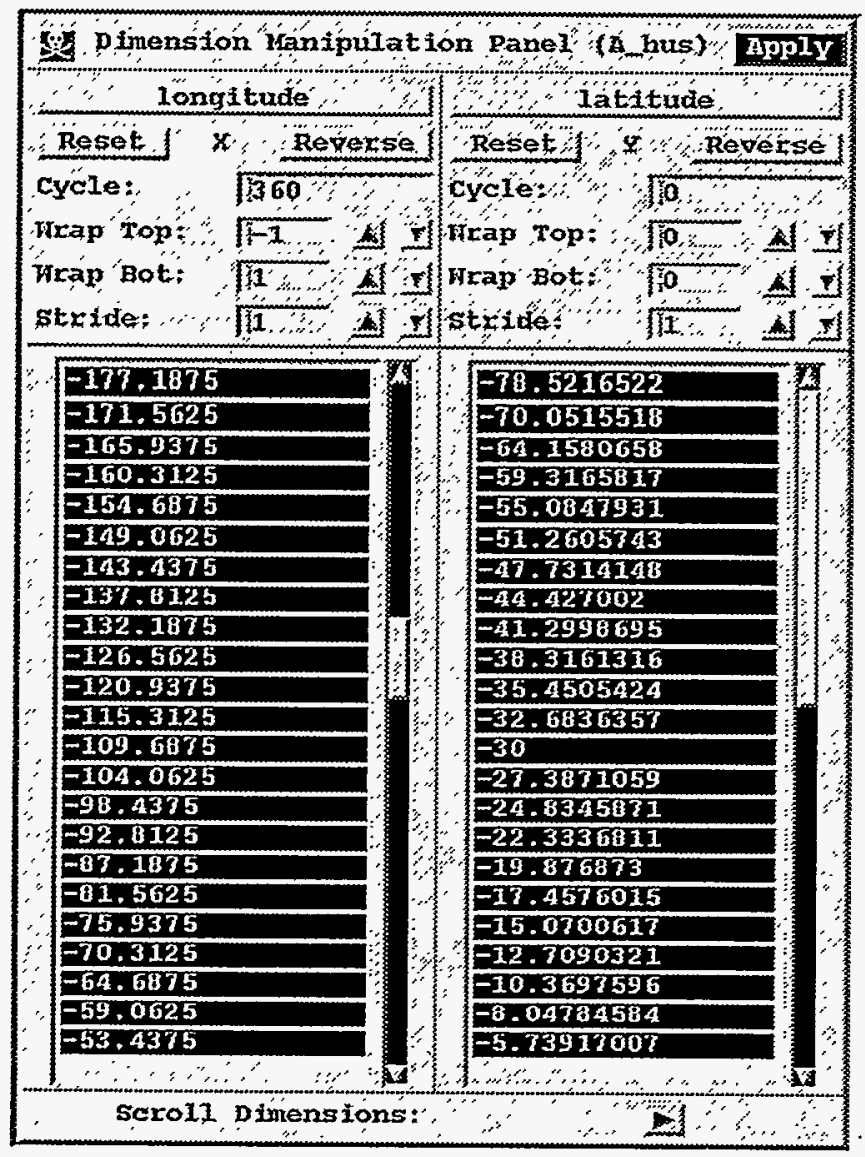

The Dimension Manipulation Panel displays a column for each dimension that is defined in the array data attribute set. This panel can be used to transpose or reverse dimensions, set the cycle value used in wrapping a dimension's top or bottom, stride a dimension's coordinate values, and reset a dimension's original values.

\section{How to Use the Dimension Manipulation Panel}

\section{Selecting Top Dimension Coordinate Value:}

The dimension scroll window lists the dimension's coordinate values. Move the pointer to the desired top value and press the left mouse button. The selected value will be highlighted.

\section{Selecting Bottom Dimension Coordinate Value:}

The dimension scroll window lists the dimension's coordinate values. Move the pointer to the desired bottom value and press the middle mouse button. The selected value will be highlighted. 


\section{Toggling Dimension Values:}

The dimension scroll window lists the dimension's coordinate values. Move the pointer to the desired value and press the right mouse button. The selected value will be highlighted. Follow the same procedure to deselect a dimension coordinate value.

\section{Transposing Dimensions:}

To transpose dimensions, move the pointer over the dimension name button (i.e., longitude, latitude, etc.) and press the left mouse button. A scroll window displaying other dimension names will appear. Move the pointer over the desired dimension name and press the left mouse button. The current dimension and the selected dimension will then be transposed.

\section{Reversing the Dimension Coordinate Values:}

Move the pointer over the 'Reverse' button and press the left mouse button. The listed coordinate values, displayed below in the scroll window, will be shown in reverse order.

\section{Setting the Dimension Cycle Value:}

The dimension cycle value is used in wrapping a dimension (see below), e.g. the longitude dimension has a cycle value of 360 (i.e., 0 and 360 are the same coordinate point). To set the cycle value, move the pointer in the 'Cycle' input text window and press the left mouse button. Then enter the desired cycle value.

Wrapping Top or Bottom Dimension Values:

The 'Wrap Top' or 'Wrap Bot' input text windows allow wrapping of a dimension multiple times prior to selecting top and bottom coordinate values. To wrap, move the pointer into the input text window and press the left mouse button. Then enter the desired value followed by a 'Return'. The arrow buttons can also be used to set the desired wrap values.

Striding the Dimension Coordinate Values:

To stride the dimension coordinate, move the pointer into the 'Stride' input text window and press the left mouse button. Enter the desired stride value followed by a 'Return'. The arrow buttons can also be used to set the desired stride.

\section{Resetting the Dimension Values:}

To reset the dimension column, move the pointer over the 'Reset' button and press the left mouse button.

Viewing Hidden Dimensions:

To view hidden dimensions, move the pointer to 'Scroll Dimensions' arrow buttons (at bottom) and press the left mouse button. The dimension columns will scroll to the left or to the right, depending on the arrow button selected. 


\subsection{Dimension Assignment Panel}

Access: via Data Editor Panel -> 'VALUES - ' button

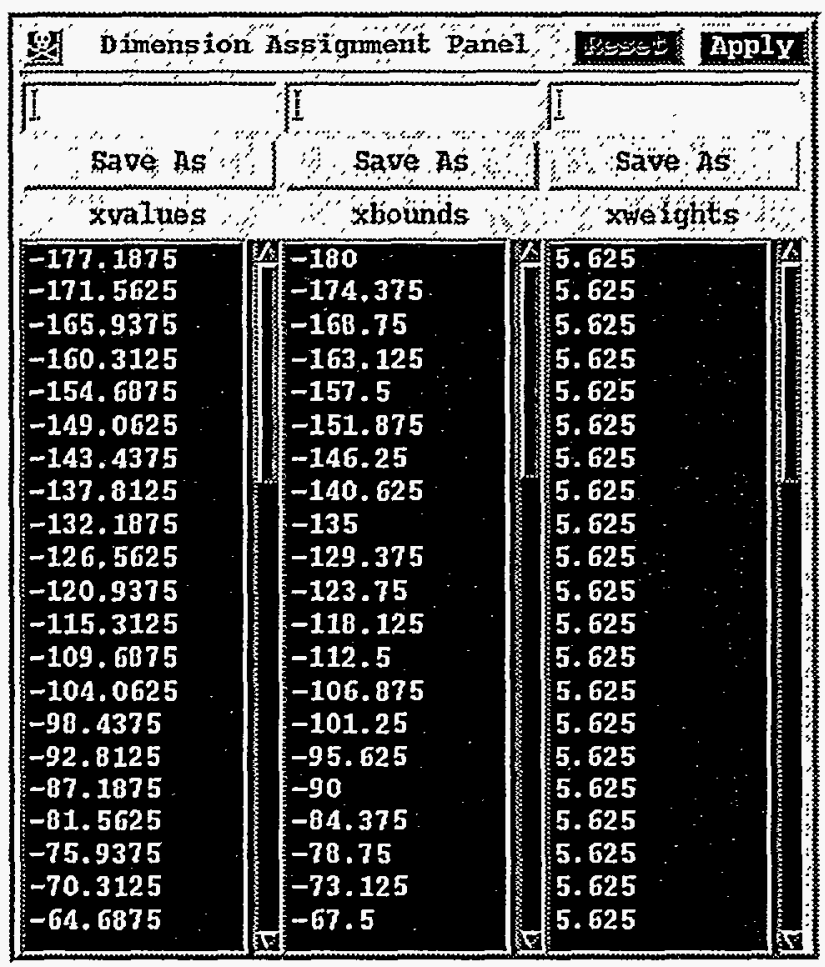

The Dimension Assignment Panel displays dimension coordinate values, bounds, and weights, and can be used to create lists that are displayed in the List Table Panel (see associated Hints). With the Dimension Assignment Panel, the data attribute set also can be transformed to a different grid.

\section{How to Use the Dimension Assignment Panel}

\section{Creating a List Attribute Set:}

Position the pointer over the 'Save As' button of the list category to be created (i.e., xvalues, xbounds, xweights, etc.) and press the left mouse button. An input text window will then appear. Enter the new list attribute set name and select 'Save' (to create the list) or 'Cancel' (to cancel the operation). The new list attribute set name will be visible in the List Table Panel (see associated Hints).

\section{Transforming a Grid:}

Access the List Table Panel (via Main Menu - 'Basic' $\rightarrow$ 'List Table'). Move the pointer over the list attribute set name of the desired grid and press the middle mouse button to copy the list. Then move the pointer to the desired yellow Dimension Assigment Panel input text window and press the middle mouse button to drop the list. Press the red 'Apply' button to view changes. 


\subsection{Boxfill Editor Panel}

Access: via Boxfill Graphics Method Panel -> Attribute Set Name

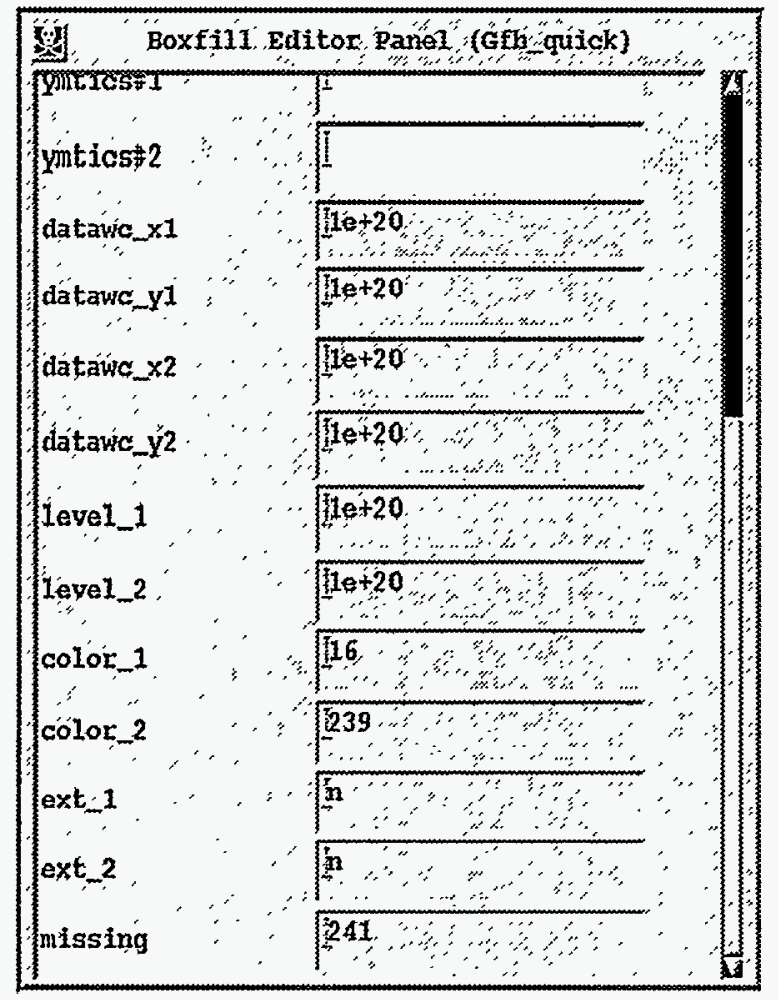

The Boxfill Editor Panel displays the boxfill graphics method attribute set names and assignment values. The boxfill graphics method displays a two-dimensional data array by surrounding each data value by a colored grid box.

\section{How to Use the Boxfill Editor Panel}

Changing the Map Projection:

Move the pointer over the 'projection' menu button and press and hold the left mouse button. Then move the pointer over the desired map projection and release the left mouse button. Press the red 'Apply' button to register changes.

\section{Setting a List Name for Labels and Tick Marks:}

Labels and tick marks are: 'xticlabels\#1', bottom prefixed list name for $\mathrm{x}$ axis labels and ticks; 'xticklabels\#2', top prefixed list name for $x$ axis labels and ticks; 'xmtics\#1, left prefixed list name for $\mathrm{x}$ axis minor ticks; $\mathrm{xmtics} \# 2$, right prefixed list name for $\mathrm{x}$ axis minor ticks; 'yticlabels\#1', left prefixed list name for y axis labels and ticks; 'yticlabels\#2', right prefixed list name for y axis labels and ticks; 'ymtics\#1', right prefixed list name for y axis minor ticks; 'ymtics\#2', left prefixed list name for $y$ axis minor ticks. From the List Table Panel, copy and drop the new attribute set name into the yellow input text window (see associated Hints for copy and drop procedures). 


\section{Defining Data Space in Real-World Coordinates:}

The data space defined in the picture template in normalized device coordinates is mapped to the real-world coordinates given: 'datawc_x1', 'datawc_x2', 'datawc_y1', 'datawc_y2'. A value of ' $1 \mathrm{e}+20$ ' cues VCS to use the data coordinate values.

\section{Setting Levels:}

Set the levels by changing 'level_1' and 'level_2' input text windows. If 'level_1' or 'level_2' is set to ' $1 \mathrm{e}+20$ ', then VCS will select the levels.

\section{Setting Colormap Range:}

Set the color range by changing 'color_1' and 'color_2' input text windows. (The colormap ranges from 0 to 255 , but only color indices 0 through 239 can be changed.) See Hints on the Colormap Editor/Table Panel for procedures to select and modify the colormaps.

\section{Showing Extended Values:}

If the minimum data value is less than the 'level_ 1 ' value and 'ext_ 1 ' is set to ' $n$ ', then all data values less than the 'level_1' value are not displayed. If 'ext_1' is set to ' $y$ ', then the values less than 'level_1' are given 'color_1' color index value and are displayed. If the maximun data value is greater than the 'level_2' value and 'ext_2' is set to ' $n$ ', then all data values greater than the 'level_2' value are not displayed. If 'ext_2' is set to ' $y$ ', then the values greater than 'level_2' are given 'color_2' color index value and are displayed.

\section{Showing Missing Data:}

The color index value for missing data is specified by setting the 'missing' input text window. The minimum color index value is 0 and the maximum color index value is 255 . See Hints associated with the Colormap Editor/Table Panel for details on changing color indices. 


\subsection{Continents Editor Panel}

Access: via Continents Graphics Method Panel -> Attribute Set Name

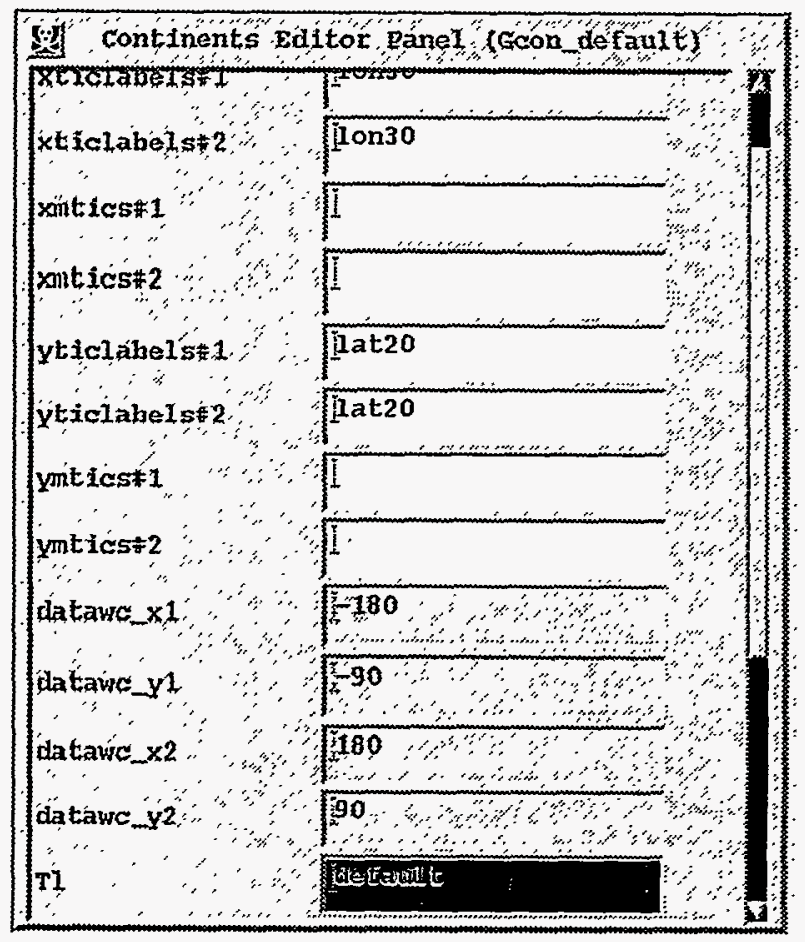

The Continents Editor Panel displays the continents graphics method attribute set names and assignment values. The continents graphics method displays a predefined, generic set of continental outlines in a longitude by latitude space.

\section{How to use the Continents Editor Panel}

Changing the Map Projection:

Move the pointer over the 'projection' menu button'and press and hold the left mouse button. Then move the pointer over the desired map projection and release the left mouse button. Press the red 'Apply' button to register changes.

\section{Setting a List Name for Labels and Tick Marks:}

Labels and tick marks are: 'xticlabels\#1', bottom prefixed list name for $\mathrm{x}$ axis labels and ticks; 'xticklabels\#2', top prefixed list name for $\mathrm{x}$ axis labels and ticks; ' $\mathrm{xmtics} \# 1$, left prefixed list name for $\mathrm{x}$ axis minor ticks; xmtics\#2, right prefixed list name for $\mathrm{x}$ axis minor ticks; 'yticlabels\#1', left prefixed list name for y axis labels and ticks; 'yticlabels\#2', right prefixed list name for y axis labels and ticks; 'ymtics\#1', right prefixed list name for y axis minor ticks; 'ymtics\#2', left prefixed list name for $y$ axis minor ticks. From the List Table Panel, copy and drop the new name into the yellow input text window ( see associated Hints for copy and drop procedures). 


\section{Defining Data Space in Real-World Coordinates:}

The data space defined in the picture template in normalized device coordinates is mapped to the real-world coordinates given: 'datawc_x1', 'datawc_x2', 'datawc_y1', 'datawc_y2'. A value of ' $1 \mathrm{e}+20$ ' cues VCS to use the data coordinate values.

\section{Changing Line Type:}

To change the continents line type, access the (Tl) Line Table Panel (via Main Menu - 'Basic' -> 'Line Table (Tl)'). To copy a line type, move the pointer to the desired line attribute set name and press the middle mouse button. To drop, move the pointer to the cyan ' $\mathrm{Tl}$ ' input text window on the Continents Editor Panel and press the middle mouse button. Press the red 'Apply' button to register changes.

\subsection{Isofill Editor Panel}

Access: via Isofill Graphics Method Panel -> Attribute Set Name

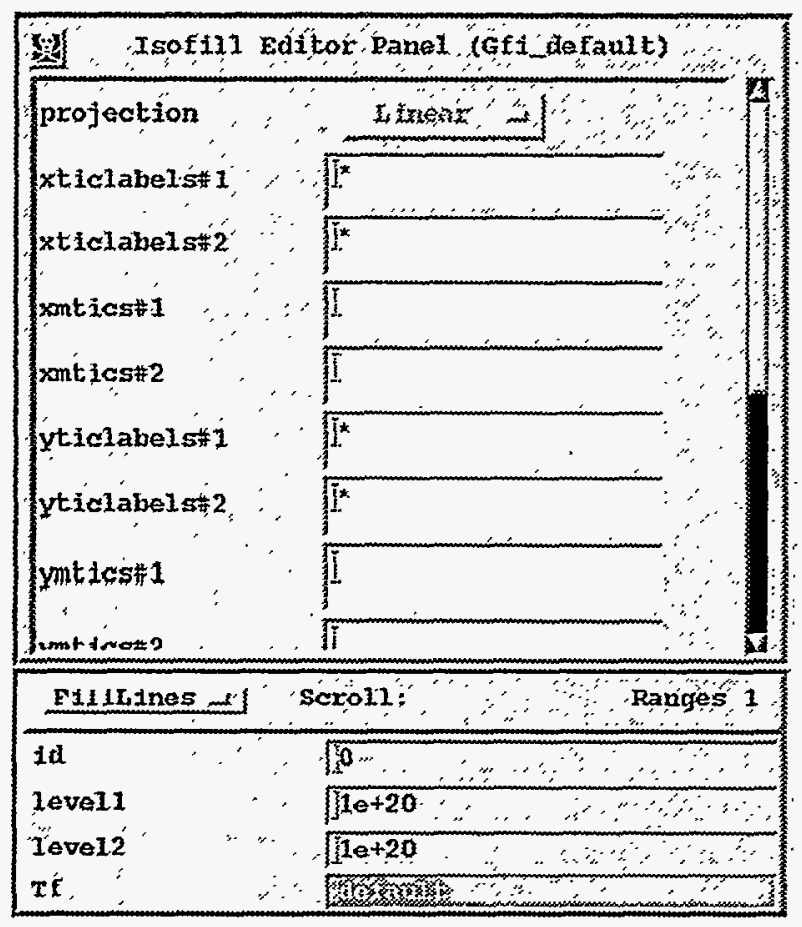

The Isofill Editor Panel displays the isofill graphics method attribute set names and assignment values. The isofill graphics method fills the area between selected isolevels (levels of constant value) of a two-dimensional array.

\section{How to Use the Isofill Editor Panel}

\section{Changing the Map Projection:}

Move the pointer over the 'projection' menu button and press and hold the left mouse button. Then move the pointer over the desired map projection and release the left mouse button. Press the red 'Apply' button to register changes. 
Setting a List Name for Labels and Tick Marks:

Labels and tick marks are: 'xticlabels\#1', bottom prefixed list name for $\mathrm{x}$ axis labels and ticks; 'xticklabels\#2', top prefixed list name for $\mathrm{x}$ axis labels and ticks; ' $x$ mtics\#1, left prefixed list name

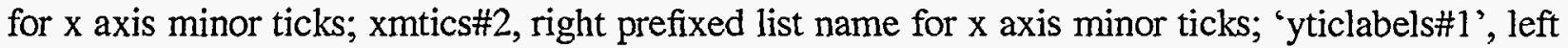
prefixed list name for y axis labels and ticks; 'yticlabels\#2', right prefixed list name for y axis labels and ticks; 'ymtics\#1', right prefixed list name for y axis minor ticks; 'ymtics\#2', left prefixed list name for y axis minor ticks. From the List Table Panel, copy and drop the new attribute set name into the yellow input text window (see associated Hints for copy and drop procedures).

\section{Defining Data Space in Real-World Coordinates:}

The data space defined in the picture template in normalized device coordinates is mapped to the real-world coordinates given: 'datawc_x1', 'datawc_x2', 'datawc_y1', 'datawc_y2'. A value of ' $1 \mathrm{e}+20$ ' cues VCS to use the data coordinate values.

\section{Setting Range Attributes:}

The Isofill Range Panel appears below the Isofill Editor Panel. It allows the creating and setting of isolevels. The 'id' input text window shows a unique integer identifier that cannot be modified. The 'level1' and 'level2' input text windows show the first and second bounding levels. Move the pointer into the corresponding window and press the left mouse button, then modify to the desired value. If a value of ' $1 \mathrm{e}+20$ ' shows in the input text window, then VCS will enter the levels. The 'Tf' input text window indicates the fillarea basic element that is in use. (See associated Hints for copy and drop procedures.) 


\subsection{Isoline Editor Panel}

Access: via Isoline Graphics Method Panel -> Attribute Set Name

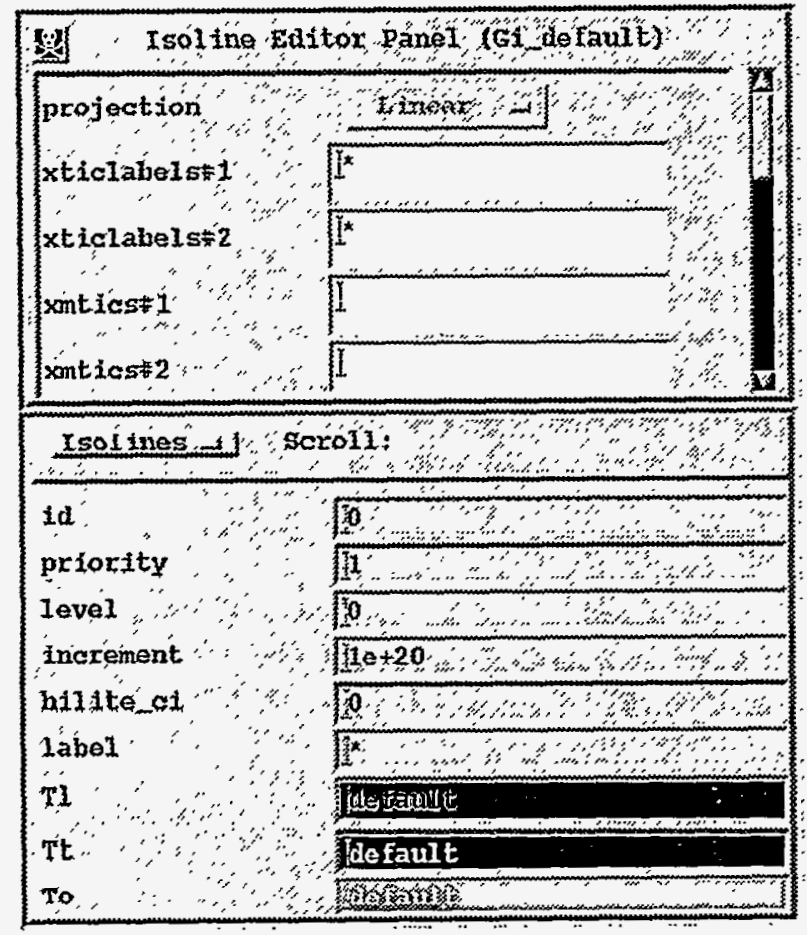

The Isoline Editor Panel displays the isoline graphics method attribute set names and assignment values. The isoline graphics method draws isolines (lines of constant value) at specified levels to graphically represent a two-dimensional array, and it also labels the values of these isolines.

\section{How to Use the Isoline Editor Panel}

\section{Changing the Map Projection:}

Move the pointer over the 'projection' menu button and press and hold the left mouse button. Then move the pointer over the desired map projection and release the left mouse button. Press the red 'Apply' button to register changes.

\section{Setting a List Name for Labels and Tick Marks:}

Labels and tick marks are: 'xticlabels\#1', bottom prefixed list name for $\mathrm{x}$ axis labels and ticks; 'xticklabels\#2', top prefixed list name for $\mathrm{x}$ axis labels and ticks; 'xmtics\#1, left prefixed list name for $\mathrm{x}$ axis minor ticks; $\mathrm{xmtics} \# 2$, right prefixed list name for $\mathrm{x}$ axis minor ticks; 'yticlabels\#1', left prefixed list name for y axis labels and ticks; 'yticlabels\#2', right prefixed list name for $y$ axis labels and ticks; 'ymtics\#1', right prefixed list name for y axis minor ticks; 'ymtics\#2', left prefixed list name for y axis minor ticks. From the List Table Panel, copy and drop the new attribute set name into the yellow input text window (see associated Hints for copy and drop procedures). 

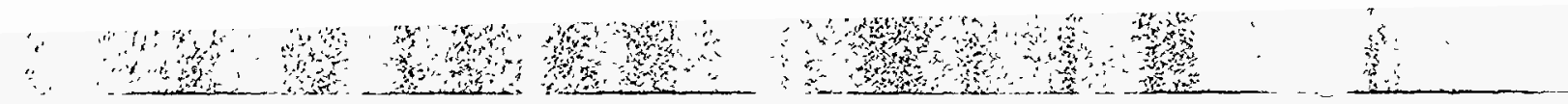

Defining Data Space in Real-World Coordinates:

The data space defined in the picture template in normalized device coordinates is mapped to the real-world coordinates given: 'datawc_x1', 'datawc_x2', 'datawc_y1', 'datawc_y2'. A value of '1e+20' cues VCS to use the data coordinate values.

\section{Displaying Isoline Labels:}

To display isoline labels, move the pointer over the 'make_labels' input text window and change the text to ' $y$ '. Select the red 'Apply' button at the top of the Isoline Editor Panel to register changes.

\section{Setting Line Attributes of Isolines:}

The Isoline Panel appears below the Isoline Editor Panel. It allows the creating and setting of line attributes of isolines. The 'id' input text window shows a unique integer identifier that cannot be modified. The 'priority' input text window shows the integer priority value, with the highest value on top. The 'level' input text window shows the starting level. The 'increment' input text window shows the increment between successive levels. The 'hilite_ci' input text window shows the color index for highlighting labels. The 'label' input text window shows the text for a label or '*' to obtain the actual level on each. The cyan ' $\mathrm{Tl}$ ' input text window shows the line attribute set name. The magenta ' $T t$ ' input text window shows the text attribute set name. The orange 'To' input text window shows the text orientation attribute set name. To modify any of these input text windows, move the pointer into the desired window and press the left mouse button. (For copy and drop procedures, see Hints associated with the (TI) Line Table Panel, and with the (Tt) Text Table and (To) Text Orientation Table Panels.) 


\subsection{Outfill Editor Panel}

Access: via Outfill Graphics Method Panel -> Attribute Set Name

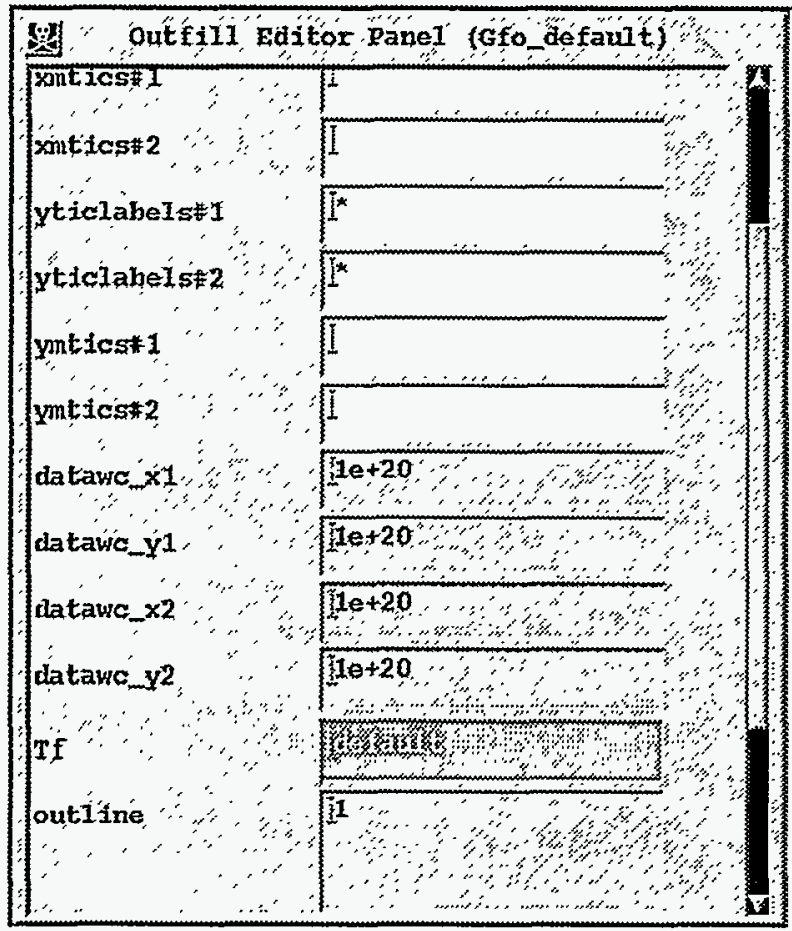

The Outfill Editor Panel displays the outfill graphics method attribute set names and assignment values. The primary purpose of the outfill graphics method is to display continents by filling their area as defined by a surface type array that indicates land, ocean, and sea-ice points. More generally, the outfill graphics method fills a set of integer values in any data array.

\section{How to Use the Outfill Editor Panel}

\section{Changing the Map Projection:}

Move the pointer over the 'projection' menu button and press and hold the left mouse button. Then move the pointer over the desired map projection and release the left mouse button. Press the red 'Apply' button to register changes.

\section{Setting a List Name for Labels and Tick Marks:}

Labels and tick marks are: 'xticlabels\#1', bottom prefixed list name for $\mathrm{x}$ axis labels and ticks; 'xticklabels\#2', top prefixed list name for $\mathrm{x}$ axis labels and ticks; 'xmtics\#1, left prefixed list name for $\mathrm{x}$ axis minor ticks; xmtics\#2, right prefixed list name for $\mathrm{x}$ axis minor ticks; '. $y$ ticlabels\#1', left prefixed list name for $y$ axis labels and ticks; 'yticlabels\#2', right prefixed list name for $y$ axis labels and ticks; 'ymtics\#1', right prefixed list name for y axis minor ticks; 'ymtics\#2', left prefixed list name for $y$ axis minor ticks. From the List Table Panel, copy and drop the new attribute set name into the yellow input text window (see associated Hints for copy and drop procedures). 


\section{Defining Data Space in Real-World Coordinates:}

The data space defined in the picture template in normalized device coordinates is mapped to the real-world coordinates given: 'datawc_x1', 'datawc_x2', 'datawc_y1', 'datawc_y2'. A value of ' $1 \mathrm{e}+20$ ' cues VCS to use the data coordinate values.

\section{Changing the Fillarea Type:}

To change the fillarea type of the outfill, access the (Tf) Fillarea Table Panel (via Main Menu 'Basic' -> 'Fillarea Table (Tf)'). To copy a fillarea type, move the pointer to the desired fillarea attribute set name and press the middle mouse button. To drop, move the pointer to the dark green 'Tf' input text window on the Outfill Editor Panel and press the middle mouse button. Press the red 'Apply' button to register changes.

\section{Setting the Outline Values:}

Move the pointer into the 'outline' input text window and press the left mouse button. Type in the desired values separated by commas ','. As few as one, or as many as ten values can be given.

\subsection{Outline Editor Panel}

Access: via Outline Graphics Method Panel -> Attribute Set Name

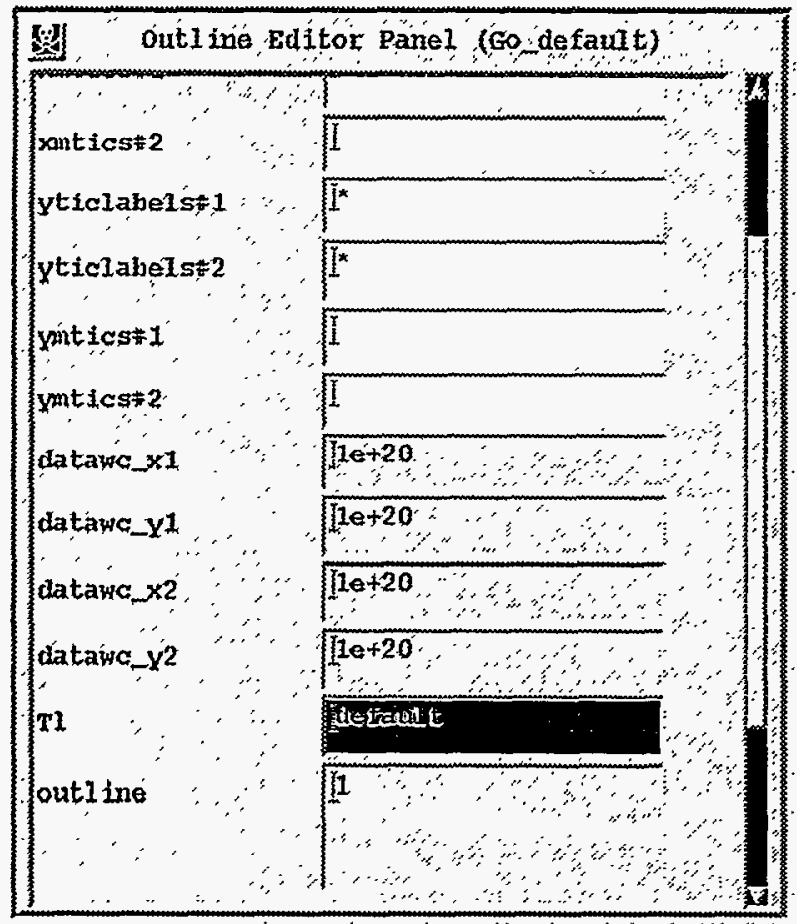

The Outline Editor Panel displays the outline's graphics method attribute set names and assignment values. The primary purpose of the outline graphics method is to display an outline of continents using a surface type array that indicates land, ocean, and sea-ice points. More generally, the outline graphics method is used to outline a set of integer values for any array. 


\section{How to Use the Outline Editor Panel}

Changing the Map Projection:

Move the pointer over the 'projection' menu button and press and hold the left mouse button. Then move the pointer over the desired map projection and release the left mouse button. Press the red 'Apply' button to register changes.

Setting a List Name for Labels and Tick Marks:

Labels and tick marks are: 'xticlabels\#1', bottom prefixed list name for $\mathrm{x}$ axis labels and ticks; 'xticklabels\#2', top prefixed list name for $\mathrm{x}$ axis labels and ticks; 'xmtics\#1, left prefixed list name for $\mathrm{x}$ axis minor ticks; $\mathrm{xmtics} \# 2$, right prefixed list name for $\mathrm{x}$ axis minor ticks; 'yticlabels\#1', left prefixed list name for y axis labels and ticks; 'yticlabels\#2', right prefixed list name for y axis labels and ticks; 'ymtics\#1', right prefixed list name for y axis minor ticks; 'ymtics\#2', left prefixed list name for y axis minor ticks. From the List Table Panel, copy and drop the new attribute set name into the yellow input text window (see associated Hints for copy and drop procedures).

\section{Defining Data Space in Real-World Coordinates:}

The data space defined in the picture template in normalized device coordinates is mapped to the real-world coordinates given: 'datawc_x1', 'datawc_x2', 'datawc_y1', 'datawc_y2'. A value of ' $1 \mathrm{e}+20$ ' cues VCS to use the data coordinate values.

\section{Changing the Line Type:}

To change the line type of the outline, access the ( $\mathrm{Tl})$ Line Table Panel (via Main Menu - 'Basic' -> 'Line Table (Tl)'). To copy a line type, move the pointer to the desired line attribute set name and press the middle mouse button. To drop, move the pointer to the cyan ' $\mathrm{Tl}$ ' input text window on the Outline Editor Panel and press the middle mouse button. Press the red 'Apply' button to register changes.

Setting the Outline Values:

Move the pointer into the 'outline' input text window and press the left mouse button. Type in the desired values separated by commas ','. As few as one, or as many as ten values can be given. 


\subsection{Scatter Editor Panel}

Access: via Scatter Graphics Method Panel -> Attribute Set Name

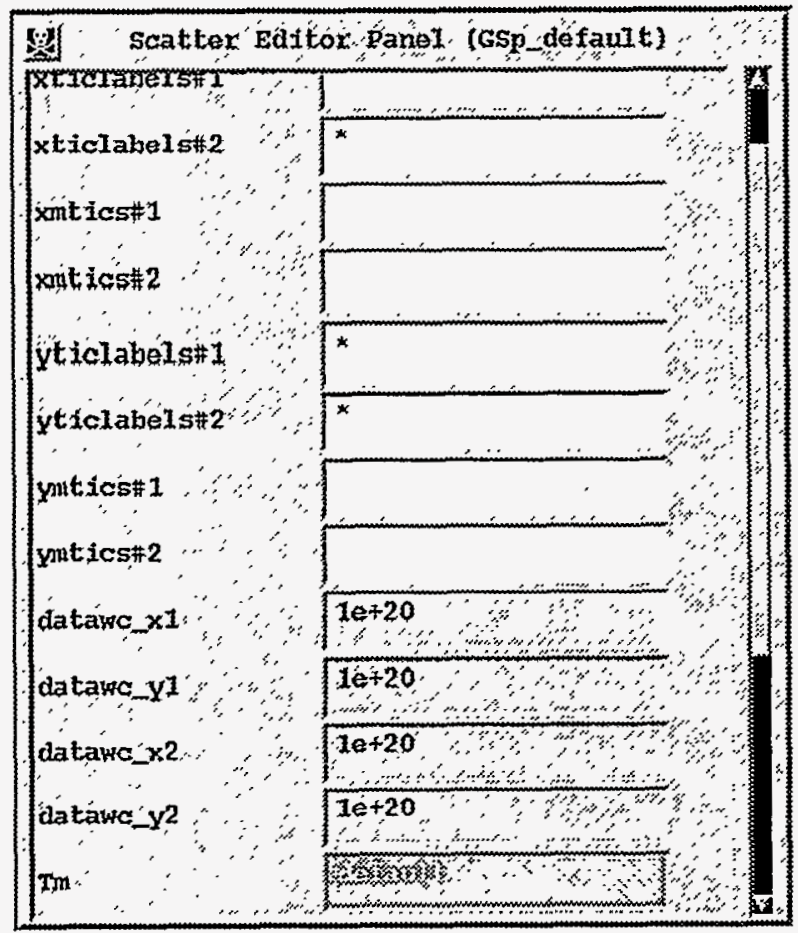

The Scatter Editor Panel displays the attribute set names and assignment values of the scatter graphics method. The scatter graphics method displays a scatter plot of two data arrays, that is $A(x, y, z, t)$ and $B(x, y, z, t)$.

\section{How to Use the Scatter Editor Panel}

\section{Changing the Map Projection:}

Move the pointer over the 'projection' menu button and press and hold the left mouse button. Then move the pointer over the desired map projection and release the left mouse button. Press the red 'Apply' button (in upper right corner) to register changes.

Setting a List Name for Labels and Tick Marks:

Labels and tick marks are: 'xticlabels\#1', bottom prefixed list name for $\mathrm{x}$ axis labels and ticks;

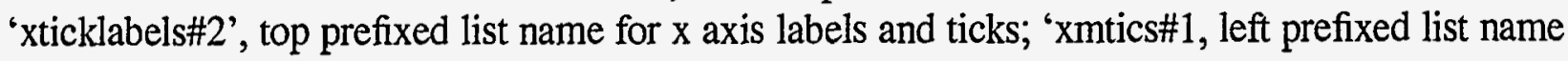
for $\mathrm{x}$ axis minor ticks; xmtics\#2, right prefixed list name for $\mathrm{x}$ axis minor ticks; 'yticlabels\#1', left prefixed list name for y axis labels and ticks; 'yticlabels\#2', right prefixed list name for y axis labels and ticks; 'ymtics\#1', right prefixed list name for y axis minor ticks; 'ymtics\#2', left prefixed list name for y axis minor ticks. From the List Table Panel, copy and drop the new attribute set name into the yellow input text window (see Hints on the List Table Panel for procedures). 


\section{Defining Data Space in Real-World Coordinates:}

The data space defined in the picture template in normalized device coordinates is mapped to the real-world coordinates given: 'datawc_x1', 'datawc_x2', 'datawc_y1', 'datawc_y2'. A value of ' $1 \mathrm{e}+20$ ' cues VCS to use the data coordinate values.

\section{Changing the Marker Type:}

To change the scatter marker type, access the (Tm) Marker Table Panel (via Main Menu - 'Basic' -> 'Marker Table (Tm)'). To copy a marker type, move the pointer to the desired marker attribute set name and press the middle mouse button. To drop, move the pointer to the brown ' $\mathrm{Tm}$ ' input text window on the Scatter Editor Panel and press the middle mouse button. Press the red 'Apply' button to register changes.

\subsection{Vector Editor Panel}

Access: via Vector Graphics Method Panel -> Attribute Set Name

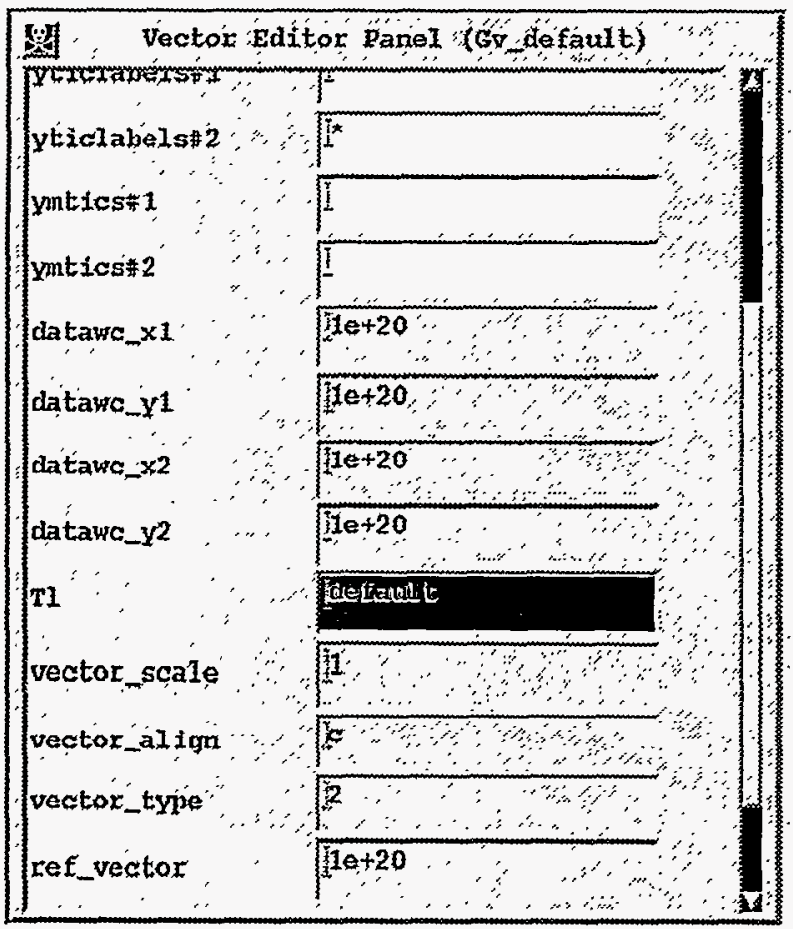

The Vector Editor Panel displays the attribute set names and assignment values of the vector graphics method. The primary purpose of the vector graphics method is to display a vector plot of a $2 \mathrm{D}$ vector field. Vectors are located at the coordinate locations and point in the direction of the data vector field. Vector magnitudes are the product of data vector field lengths and a scaling factor. 


\section{How to Use the Vector Editor Panel}

\section{Changing the Map Projection:}

Move the pointer over the 'projection' menu button and press and hold the left mouse button. Then move the pointer over the desired map projection and release the left mouse button. Press the red 'Apply' button (in upper right corner) to register changes.

Setting a List Name for Labels and Tick Marks:

Labels and tick marks are: 'xticlabels\#1', bottom prefixed list name for $\mathrm{x}$ axis labels and ticks; 'xticklabels\#2', top prefixed list name for $\mathrm{x}$ axis labels and ticks; 'xmtics\#1, left prefixed list name for $\mathrm{x}$ axis minor ticks; xmtics\#2, right prefixed list name for $\mathrm{x}$ axis minor ticks; 'yticlabels\#1', left prefixed list name for $y$ axis labels and ticks; 'yticlabels\#2', right prefixed list name for y axis labels and ticks; 'ymtics\#1', right prefixed list name for y axis minor ticks; 'ymtics\#2', left prefixed list name for y axis minor ticks. From the List Table Panel, copy and drop the new attribute set name into the yellow input text window (see associated Hints for copy and drop procedures).

\section{Defining Data Space in Real-World Coordinates:}

The data space defined in the picture template in normalized device coordinates is mapped to the real-world coordinates given: 'datawc_x1', 'datawc_x2', 'datawc_y1', 'datawc_y2'. A value of ' $1 \mathrm{e}+20$ ' cues VCS to use the data coordinate values.

\section{Changing the Line Type:}

To change the vector line type, access the (Tl) Line Table Panel (via Main Menu - 'Basic' -> 'Line Table (Tl)'). To copy a line type, move the pointer to the desired line attribute set name and press the middle mouse button. To drop, move the pointer to the cyan ' $\mathrm{Tl}$ ' input text window on the Vector Editor Panel and press the middle mouse button. Press the red 'Apply' button to register changes.

Setting the Vector Scale Value:

Move the pointer to the 'vector_scale' input text window and press the left mouse button. Enter the desired vector scale value.

Setting the Vector Alignment:

Move the pointer to the 'vector_align' input text window and press the left mouse button. Enter: ' $h$ ', head at point; 'c', center at point; or ' $t$ ', tail at point.

Setting the Vector Type:

Move the pointer to the 'vector_type' input text window and press the left mouse button. Enter: ' 1 ', wind barbs; '2', arrow at head; '3', arrow at tail; or '4', arrow at head and tail. If a number greater than 4 is entered, wind barbs are displayed.

\section{Setting the Vector Reference:}

The vector reference defines the size of the vector in response to the data. Move the pointer to the 'ref-vector' input text window and press the left mouse button. Then enter the desired reference value. (A value of '1.e+20' cues VCS to select the reference vector.) Press the red 'Apply' button to register changes. 


\subsection{XvsY Editor Panel}

Access: via XvsY Graphics Method Panel -> Attribute Set Name

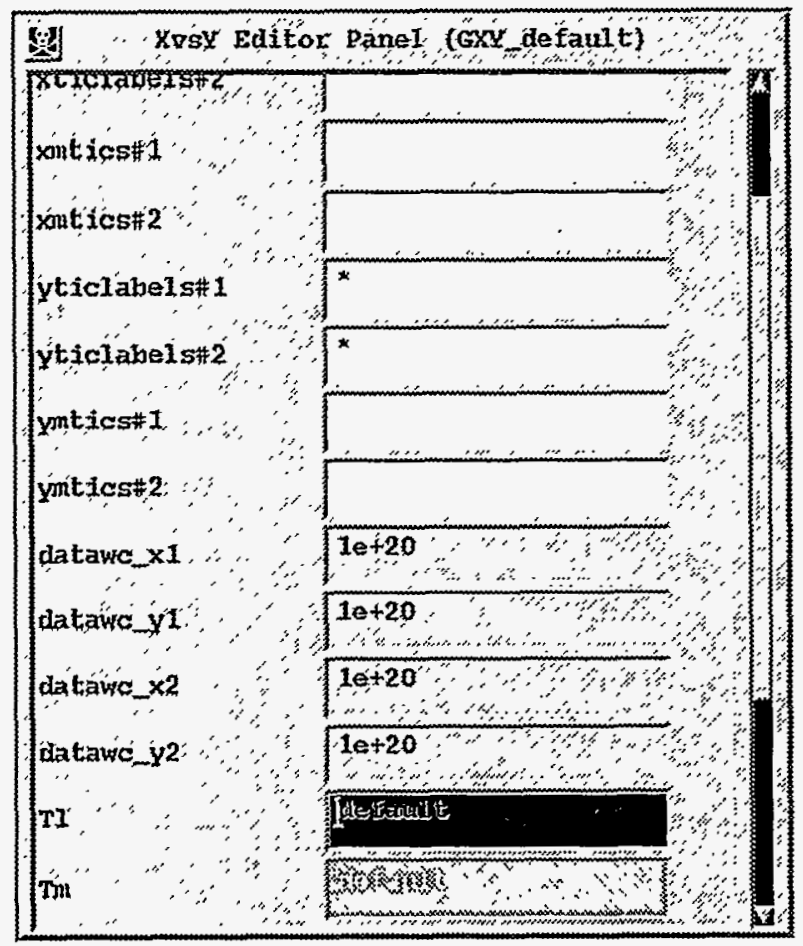

The XvsY Editor Panel displays the attribute set names and assignment values for the XvsY graphics method. The XvsY graphics method displays a line plot from two 1D data arrays, that is $X(t)$ and $Y(t)$, where $t$ represents the $1 D$ coordinate values.

\section{How to Use the XvsY Editor Panel}

\section{Changing the Map Projection:}

Move the pointer over the 'projection' menu button and press and hold the left mouse button. Then move the pointer over the desired map projection and release the left mouse button. Press the red 'Apply' button (in upper right corner) to register changes.

\section{Setting a List Name for Labels and Tick Marks:}

Labels and tick marks are: 'xticlabels\#1', bottom prefixed list name for $\mathrm{x}$ axis labels and ticks; 'xticklabels\#2', top prefixed list name for $\mathrm{x}$ axis labels and ticks; 'xmtics\#1, left prefixed list name for $\mathrm{x}$ axis minor ticks; $\mathrm{xmtics} \# 2$, right prefixed list name for $\mathrm{x}$ axis minor ticks; 'yticlabels\#1', left prefixed list name for y axis labels and ticks; 'yticlabels\#2', right prefixed list name for y axis labels and ticks; 'ymtics\#1', right prefixed list name for y axis minor ticks; 'ymtics\#2', left prefixed list name for y axis minor ticks. From the List Table Panel, copy and drop the new attribute set name into the yellow input text window (see associated Hints for copy and drop procedures). 
Defining Data Space in Real-World Coordinates:

The data space defined in the picture template in normalized device coordinates is mapped to the real-world coordinates given: 'datawc_x1', 'datawc_x2', 'datawc_y1', 'datawc_y2'. A value of ' $1 \mathrm{e}+20$ ' cues VCS to use the data coordinate values.

Changing the Line Type:

To change the XvsY line type, access the (Tl) Line Table Panel (via Main Menu - 'Basic' -> 'Line Table (Tl)'). To copy a line type, move the pointer to the desired line attribute set name and press the middle mouse button. To drop, move the pointer to the cyan ' $\mathrm{Tl}$ ' input text window of the XvsY Editor Panel and press the middle mouse button. Press the red 'Apply' button to register changes.

Changing the Marker Type:

To change the XvsY marker type, access the (Tm) Marker Table Panel (via Main Menu - 'Basic' -> 'Marker Table (Tm)'). To copy a marker type, move the pointer to the desired marker attribute set name and press the middle mouse button. To drop, move the pointer to the brown 'Tm' input text window on the XvsY Editor Panel and press the middle mouse button. Press the red 'Apply' button to register changes. 


\subsection{Xyvsy Editor Panel}

Access: via Xyvsy Graphics Method Panel -> Attribute Set Name

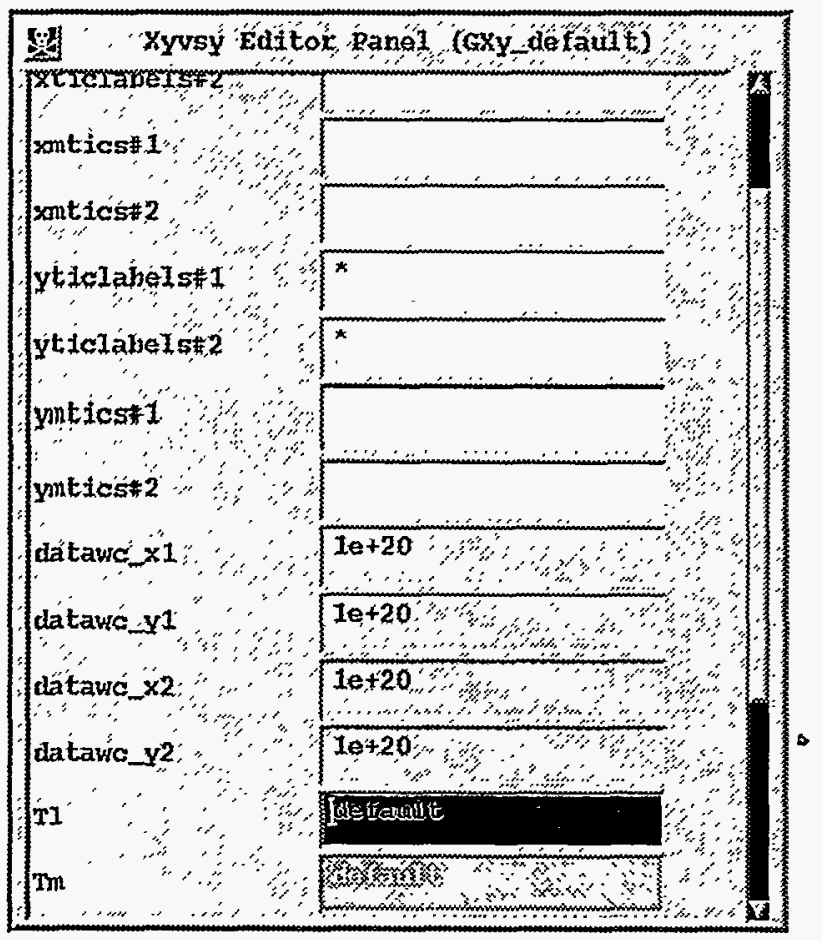

The Xyvsy Editor Panel displays the attribute set names and assignment values of the Xyvsy graphics method. The Xyvsy graphics method displays a line plot from a 1D data array, that is $\mathrm{X}(\mathrm{y})$, where $\mathrm{y}$ represents the $1 \mathrm{D}$ coordinate values.

\section{How to Use the Xyvsy Editor Panel}

\section{Changing the Map Projection:}

Move the pointer over the 'projection' menu button and press and hold the left mouse button. Then move the pointer over the desired map projection and release the left mouse button. Press the red 'Apply' button to register changes.

\section{Setting a List Name for Labels and Tick Marks:}

Labels and tick marks are: 'xticlabels\#1', bottom prefixed list name for $\mathrm{x}$ axis labels and ticks; 'xticklabels\#2', top prefixed list name for $\mathrm{x}$ axis labels and ticks; 'xmtics\#1, left prefixed list name for $\mathrm{x}$ axis minor ticks; $\mathrm{xmtics}$ 2, right prefixed list name for $\mathrm{x}$ axis minor ticks; 'yticlabels\#1', left prefixed list name for y axis labels and ticks; 'yticlabels\#2', right prefixed list name for y axis labels and ticks; 'ymtics\#1', right prefixed list name for y axis minor ticks; 'ymtics\#2', left prefixed list name for y axis minor ticks. From the List Table Panel, copy and drop the new attribute set name into the yellow input text window (see associated Hints for copy and drop procedures). 
Defining Data Space in Real-World Coordinates:

The data space defined in the picture template in normalized device coordinates is mapped to the real-world coordinates given: 'datawc_x1', 'datawc_x2', 'datawc_y1', 'datawc_y2'. A value of ' $1 \mathrm{e}+20$ ' cues VCS to use the data coordinate values.

Changing the Line Type:

To change the Xyvsy line type, access the (Tl) Line Table Panel (via Main Menu - 'Basic' -> 'Line Table ( $\mathrm{Tl})$ '). To copy a line type, move the pointer to the desired line attribute set name and press the middle mouse button. To drop, move the pointer to the cyan ' $\mathrm{Tl}$ ' input text window of the Xyvsy Editor Panel and press the middle mouse button. Press the red 'Apply' button to register changes.

\section{Changing the Marker Type:}

To change the Xyvsy marker type, access the (Tm) Marker Table Panel (via Main Menu - 'Basic' -> 'Marker Table (Tm)'). To copy a marker type, move the pointer to the desired marker attribute set name and press the middle mouse button. To drop, move the pointer to the brown ' $\mathrm{Tm}$ ' input text window on the Xyvsy Editor Panel and press the middle mouse button. Press the red 'Apply' button to register changes. 


\subsection{Yxvsx Editor Panel}

Access: via Yxvsx Graphics Method Panel -> Attribute Set Name

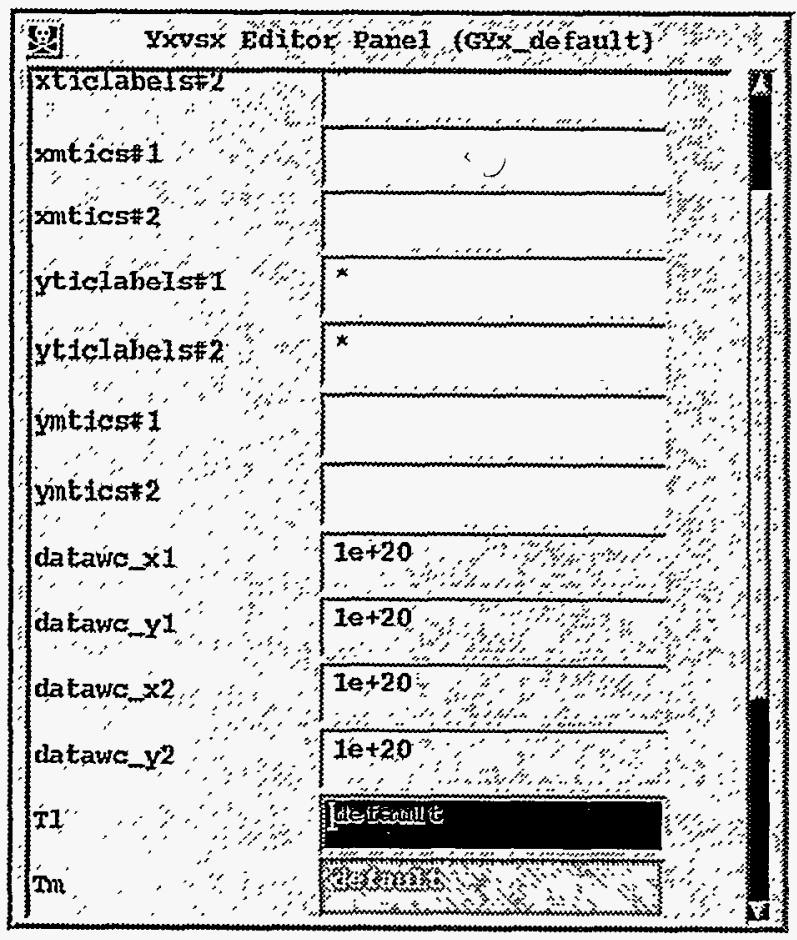

The Yxvsx Editor Panel displays the attribute set names and assignment values of the Yxvsx graphics method. The Yxvsx graphics method displays a line plot from a 1D data array, that is $\mathrm{Y}(\mathrm{x})$, where $\mathrm{x}$ represents the $1 \mathrm{D}$ coordinate values.

\section{How to Use the Yxvsx Editor Panel}

\section{Changing the Map Projection:}

Move the pointer over the 'projection' menu button and press and hold the left mouse button. Then move the pointer over the desired map projection and release the left mouse button. Press the red 'Apply' button to register changes.

Setting a List Name for Labels and Tick Marks:

Labels and tick marks are: 'xticlabels\#1', bottom prefixed list name for $\mathrm{x}$ axis labels and ticks; 'xticklabels\#2', top prefixed list name for $\mathrm{x}$ axis labels and ticks; 'xmtics\#1, left prefixed list name for $\mathrm{x}$ axis minor ticks; $\mathrm{xmtics} 2$, right prefixed list name for $\mathrm{x}$ axis minor ticks; 'yticlabels\#1', left prefixed list name for y axis labels and ticks; 'yticlabels\#2', right prefixed list name for y axis labels and ticks; 'ymtics\#1', right prefixed list name for y axis minor ticks; 'ymtics\#2', left prefixed list name for $y$ axis minor ticks. From the List Table Panel, copy and drop the new attribute set name into the yellow input text window (see associated Hints for copy and drop procedures). 


\section{Defining Data Space in Real-World Coordinates:}

The data space defined in the picture template in normalized device coordinates is mapped to the real-world coordinates given: 'datawc_x1', 'datawc_x2', 'datawc_y1', 'datawc_y2'. A value of ' $1 \mathrm{e}+20$ ' cues VCS to use the data coordinate values.

Changing the Line Type:

To change the Yxvsx line type, access the (Tl) Line Table Panel (via Main Menu - 'Basic' -> 'Line Table ( $\mathrm{Tl})$ '). To copy a line type, move the pointer to the desired line attribute set name and press the middle mouse button. To drop, move the pointer to the cyan ' $\mathrm{Tl}$ ' input text window on the Yxvsx Editor Panel and press the middle mouse button. Press the red 'Apply' button to register changes.

\section{Changing the Marker Type:}

To change the Yxvsx marker type, access the (Tm) Marker Table Panel (via Main Menu - 'Basic' $\rightarrow>$ 'Marker Table (Tm)'). To copy a marker type, move the pointer to the desired marker attribute set name and press the middle mouse button. To drop, move the pointer to the brown 'Tm' input text window on the Yxvsx Editor Panel and press the middle mouse button. Press the red 'Apply' button to register changes.

\subsection{Line Editor Panel}

Access: via (Tl) Line Table Panel -> Attribute Set Name

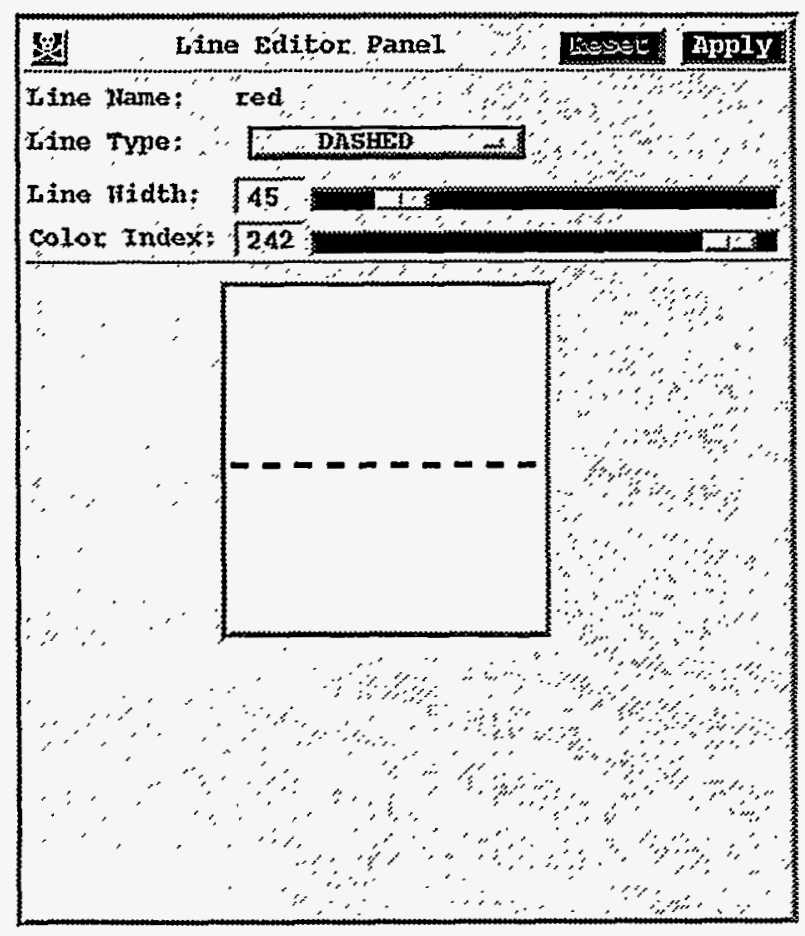

The Line Editor Panel allows editing of line attributes, including line type, width, and color index. 


\section{How to Use the Line Editor Panel}

Changing the Line Type:

Position the pointer over the 'Line Type' menu button and press and hold the left mouse button. A list of five line types will appear. Move the pointer over the desired line type and release the left mouse button. The change will appear on the canvas below. Press the red 'Apply' button to register changes.

\section{Changing the Line Width:}

Position the pointer over the 'Line Width' slider bar. While holding down the left mouse button, move the slider bar. The 'Line Width' input text window will change, and the canvas below will show the changes in line width. Press the red 'Apply' button to register changes.

\section{Changing the Color Index:}

Position the pointer over the 'Color Index' slide bar button. While holding down the left mouse button, move the slide bar button. The 'Color Index' input text window will change, and the canvas below will show the changes in color index. Press the red 'Apply' button to register changes.

\subsection{Text Editor Panel}

Access: via (Tt) Text Table Panel -> Attribute Set Name

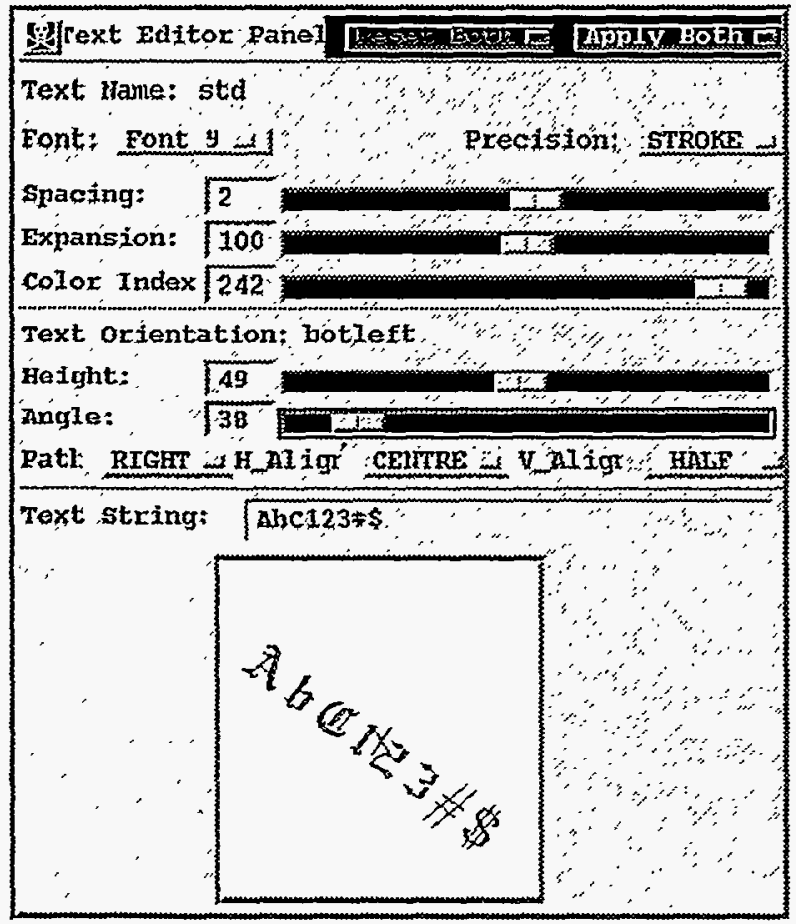

The Text Editor Panel allows editing of text attributes or text orientation attributes. Note however, the Text Editor will not allow editing of the '(Tt $)$ ' 'default' text attributes or of the '(To)' 'default', 'defcenter', 'defright', 'defcentdown', or 'defcentup' text orientation attributes. 


\section{How to Use the Text Editor Panel}

Changing the Font:

There are nine different font types. Move the pointer to the 'Font' menu button and hold down the left mouse button. Then move the pointer to the desired font and release the left mouse button. The change will appear on the canvas below. Press the red 'Apply' button to register changes. Note: if the text attribute set name is 'default', no change will occur.

Changing the Text Spacing:

Move the pointer over the 'Spacing' slider bar and press and hold down the left mouse button. Then move the slider bar to the desired location; the text displayed on the canvas below will change accordingly. The desired number can also be entered in the 'Spacing' input text window. Note: if the text attribute set name is 'default', no change will occur.

\section{Changing the Character Expansion:}

Move the pointer over the 'Expansion' slider bar and press and hold down the left mouse button. Then move the slider bar to the desired location; the text displayed on the canvas below will change accordingly. The desired number can also be entered in the 'Expansion' input text window. Note: if the text attribute set name is 'default', no change will occur.

\section{Changing the Text Color:}

Move the pointer over the 'Color Index' slider bar and press and hold down the left mouse button. Then move the slider bar to the desired location; the text displayed on the canvas below will change accordingly. The desired number can also be entered in the 'Color Index' input text window. Note: if the text attribute set name is 'default', no change will occur:

Changing the Character Height:

Move the pointer over the 'Character Height' slider bar and press and hold down the left mouse button. Then move the slider bar to the desired location; the text displayed on the canvas below will change accordingly. The desired number can also be entered in the 'Character Height' input text window. Note: if the text orientation attribute set name is 'default', 'defcenter', 'defright', 'defcentdown', or 'defcentup', no change will occur.

\section{Changing the Text Angle:}

Move the pointer over the 'Text Angle' slider bar and press and hold down the left mouse button. Then move the slider bar to the desired location; the text displayed on the canvas below will change accordingly. The desired number can also be entered in the 'Text Angle' input text window. Note: if the text orientation attribute set name is 'default', 'defcenter', 'defright', 'defcentdown', or 'defcentup', no change will occur.

\section{Changing the Text Path:}

There are four different text path selections. Move the pointer to the 'Path' menu button and hold down the left mouse button. Then move the pointer to the desired text path name and release the left mouse button. The canvas below will change to show the selected text path. Note: if the text orientation attribute set name is 'default', 'defcenter', 'defright', 'defcentdown', or 'defcentup', no change will occur. 


\section{Changing the Horizontal Alignment:}

There are three different horizontal alignment selections. Move the pointer to the ' $\mathrm{H} \_$Align' menu button and hold down the left mouse button. Then move the pointer to the desired horizontal alignment name and release the left mouse button. The canvas below will change to show the selected horizontal alignment. Note: if the text orientation attribute set name is 'default', 'defcenter', 'defright', 'defcentdown', or 'defcentup', no change will occur.

\section{Changing the Vertical Alignment:}

There are five different vertical alignment selections. Move the pointer to the ' $\mathrm{V}$ _Align' menu button and hold down the left mouse button. Then move the pointer to the desired vertical alignment name and release the left mouse button. The canvas below will change to show the selected vertical alignment. Note: if the text orientation attribute set name is 'default', 'defcenter', 'defright', 'defcentdown', or 'defcentup', no change will occur.

\subsection{Marker Editor Panel}

Access: via (Tm) Marker Table Panel -> Attribute Set Name

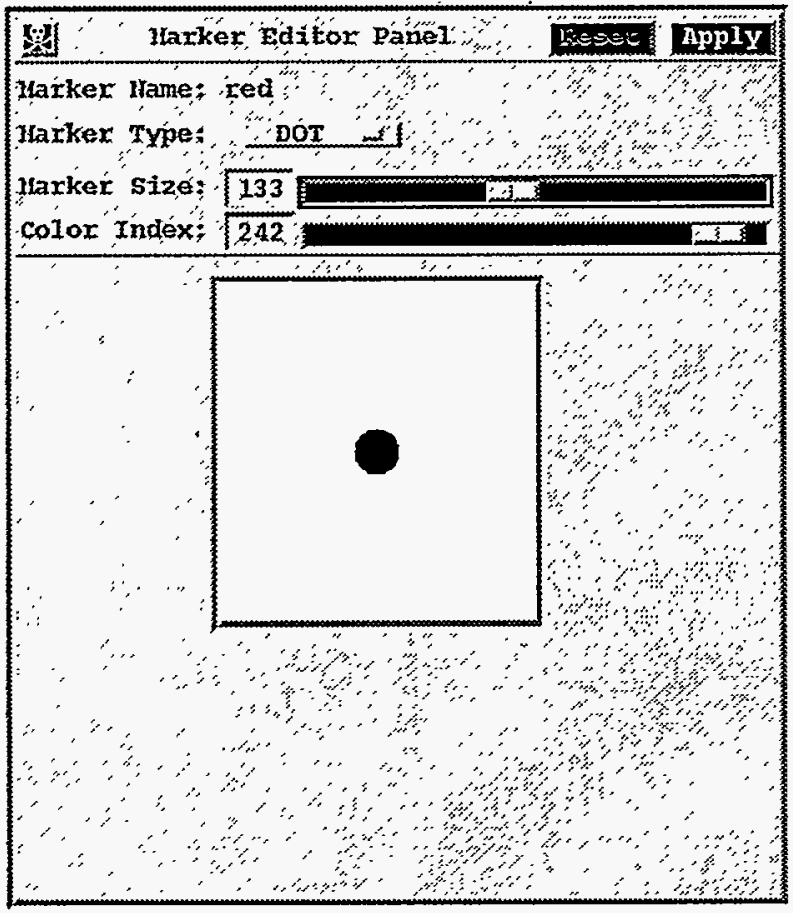

The Marker Editor Panel allows the editing of marker attributes, including marker type, width, and color index. 


\section{How to Use the Marker Editor Panel}

Changing the Marker Type:

Position the pointer over the 'Marker Type' menu button and press and hold the left mouse button. A list of five marker types will appear. Move the pointer over the desired marker type and release the left mouse button. The change will appear on the canvas below. Press the red 'Apply' button to register changes.

\section{Changing the Marker Width:}

Position the pointer over the 'Marker Width' slidee bar. While holding down the left mouse button, move the slider bar. The 'Marker Width' input text window will change when the slider bar is moved, and the canvas below will show the changes. Press the red 'Apply' button to register changes.

\section{Changing the Color Index:}

Position the pointer over the 'Color Index' slider bar. While holding down the left mouse button, move the slider bar. The 'Color Index' input text window will change when the slider bar is moved, and the canvas below will show the changes. Press the red 'Apply' button to register changes.

\subsection{Fillarea Editor Panel}

Access: via (Tf) Fillarea Table Panel -> Attribute Set Name

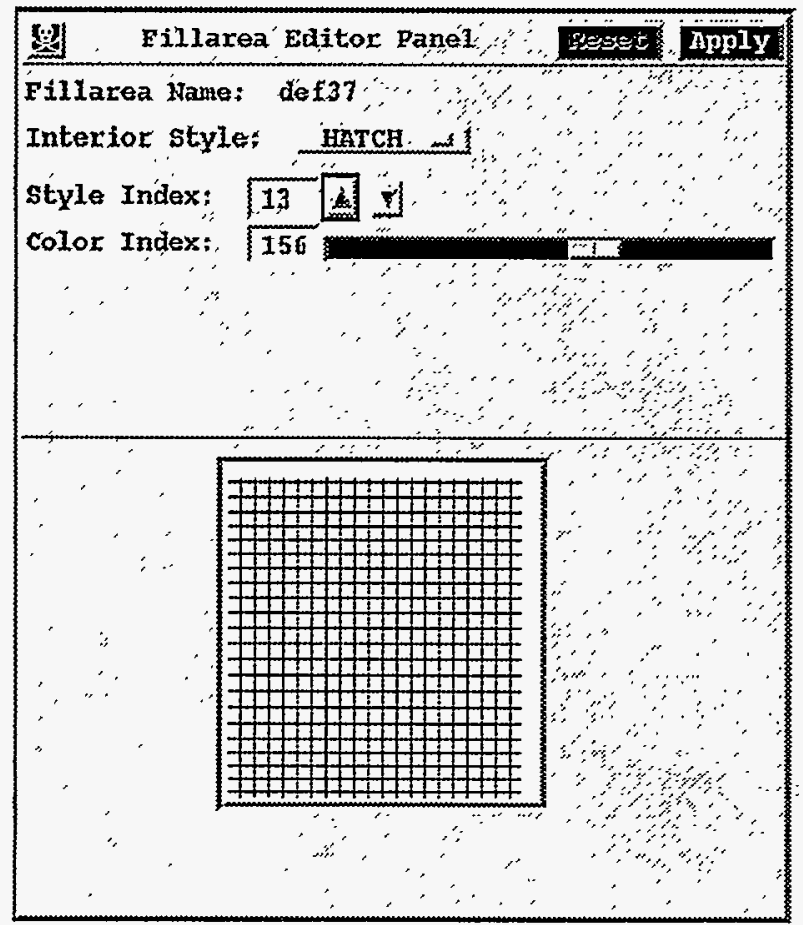

The Fillarea Editor Panel allows the user to edit fillarea attributes, including fillarea interior style, style index, and color index. Note however, the 'default' fillarea attributes cannot be edited. 


\section{How to Use the Fillarea Editor Panel}

Changing the Fillarea Interior Style:

Move the pointer over the 'Interior Style' menu button and press and hold down the left mouse button. Move the pointer over the desired interior style (i.e., 'SOLID', 'PATTERN', or 'HATCH') and release the left mouse button. The canvas below will change accordingly. Press the red 'Apply' button to register changes.

Changing the Fillarea Style Index:

The 'Style Index' is only used when the 'Interior Style' menu button is showing 'PATTERN', or 'HATCH'. Move the pointer over the up-arrow or down-arrow and press and release the left

mouse button. The canvas below will change to the appropriate style index. Press the red 'Apply' button to register changes.

Changing the Fillarea Color Index:

The 'Color Index' is only used when the 'Interior Style' menu button is showing 'SOLID', or 'HATCH'. Move the pointer over the 'Color Index' slider bar and press and hold the left mouse button. Move the slider bar to the desired color index. The changes will be shown on the canvas below. Press the red 'Apply' button to register changes. 


\subsection{Format Editor Panel}

Access: via (Th) Format Table Panel -> Attribute Set Name

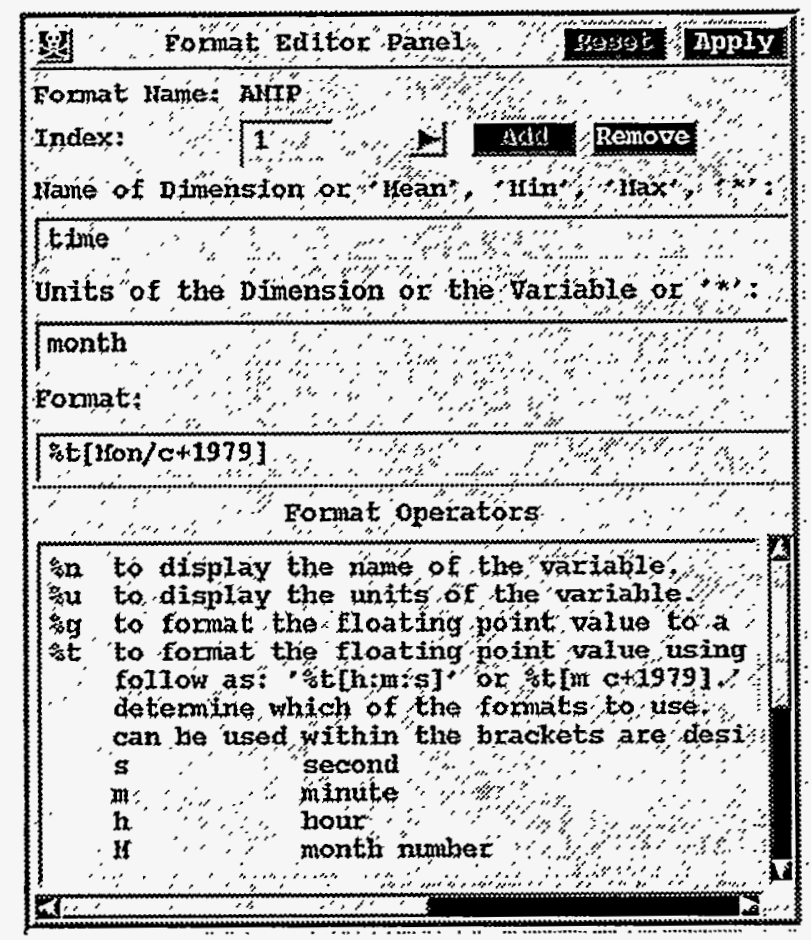

The Format Editor Panel allows editing of format attributes of a dimension, including its name, units, and format string. Note however, the 'default' format attributes cannot be edited.

\section{How to Use the Format Editor Panel}

Indexing the Format:

Move the pointer over the right arrow and press and release the left mouse button. The 'Index' input text window will increment by 1 and the Format Editor Panel will change accordingly. The 'Index' input text window can also be edited. Press the red 'Apply' button to register changes.

\section{Adding or Removing a Format Attribute:}

To add a format attribute, move the pointer over the green 'Add' button and press and release the left mouse button. To remove a format attribute, move the pointer over the red 'Remove' button and press and release the left mouse button. In both cases, the Format Editor Panel will change accordingly. Press the red 'Apply' button to register changes.

\section{Editing the Dimension Name:}

Move the pointer in the 'Name of Dimension ...' input text window and press and release the left mouse button. Enter the name of the single-valued dimension of the array data, 'Mean', 'Max', 'Min', or '*'. Press the red 'Apply' button to register changes. 
Editing the Dimension Units:

Move the pointer in the 'Units of the Dimension ...' input text window and press and release the left mouse button. Enter the unit name, variable name, or '*'. Press the red 'Apply' button to register changes.

Editing the Format String:

Move the pointer in the 'Format' input text window and press and release the left mouse button. Enter the desired format string. The format operators are listed below in the 'Format Operators' scroll window. Press the red 'Apply' button to register changes.

\subsection{List Editor Panel}

Access: via List Table Panel -> Attribute Set Name

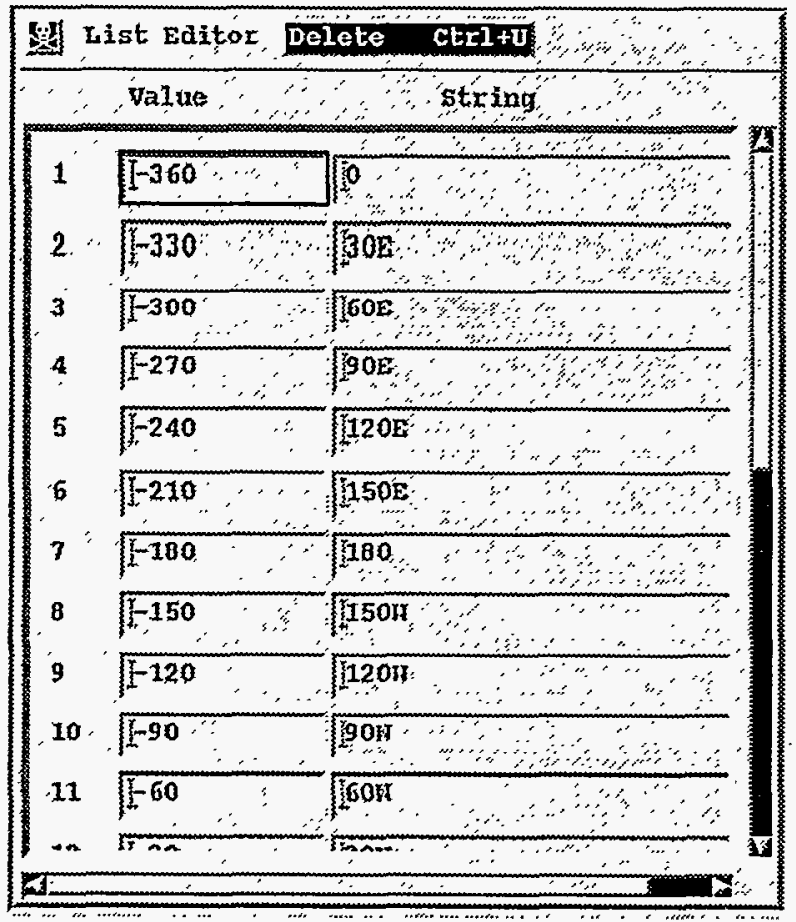

The List Editor Panel allows editing of the list attributes that define tick mark positions and dimension labels and positions. (A list consists of sequential pairs of [value, string] combinations, which can be referred to in their entirety or as a single, indexed pair.) Note however, the 'lon 30 ' and 'lat20' lists cannot be edited.

\section{How to Use the List Editor Panel}

Deleting a Line:

Move the pointer to the line to be deleted. Press and release the left mouse button in the 'Value' or 'String' input text window. From the keyboard, press the 'Ctrl' key and the ' $U$ ' key simultaneously. 
As an alternative, the red 'Delete ...' button can be selected by pressing and releasing the left mouse button. Press the red 'Apply' button to register changes.

\section{Editing a List:}

Move the pointer to the desired input text window and press and release the left mouse button. Press the red 'Apply' button to register changes.

Creating a New Line:

If the input text cursor is at the beginning of the 'Value' text and the 'Return' or 'Enter' key is pressed, then a new line will be added above the current list line. If the input text cursor is at the end of the 'String' text and the 'Return' or 'Enter' key is pressed, then a new line will be added below the current list line. Press the red 'Apply' button to register changes.

\section{Using the Arrow Keys:}

The left-arrow key moves the input text cursor one space to the left. The right-arrow key moves the input text cursor one space to the right. The up-arrow key moves the input text cursor up one list line. The down-arrow key moves the input text cursor down one list line. 


\section{Acknowledgments}

This work was performed under the auspices of the U.S. Department of Energy, Environmental Sciences Division, by the Lawrence Livermore National Laboratory under Contract W-7405-ENG-48. 